\title{
Dynamics of Knowledge Creation and Transfer: The Two Person Case*
}

\author{
Marcus Berliant** and Masahisa Fujita ${ }^{ \pm}$ \\ June 8, 2006
}

\begin{abstract}
This paper presents a micro-model of knowledge creation and transfer for a couple. Our model incorporates two key aspects of the cooperative process of knowledge creation: (i) heterogeneity of people in their state of knowledge is essential for successful cooperation in the joint creation of new ideas, while (ii) the very process of cooperative knowledge creation affects the heterogeneity of people through the accumulation of knowledge in common. The model features myopic agents in a pure externality model of interaction. In the two person case, we show that the equilibrium process tends to result in the accumulation of too much knowledge in common compared to the most productive state. Equilibrium paths are found analytically, and they are a discontinuous function of initial heterogeneity. JEL Classification Numbers: D83, O31, R11
\end{abstract}

${ }^{*}$ This research owes much to the kind hospitality and stimulating environment of the Kyoto Institute of Economic Research, where most of our arguments and do-si-do's occurred. The first author is grateful for funding from the Institute, from Washington University in St. Louis, and from the American Philosophical Society. The second author is grateful for Grants Aid for COE Research 09CE2002 and Scientific Research Grant S 13851002 from the Japanese Ministry of Education and Science. We thank Alex Anas, Gilles Duranton, Bob Hunt, Koji Nishikimi, Diego Puga, Tony Smith, Takatoshi Tabuchi, and seminar participants at Washington University, New York University, the Spring 2004 Midwest Economic Theory and International Trade conference, the Federal Reserve Bank of Philadelphia, and the 2004 North American Summer Meetings of the Econometric Society for helpful comments. Evidently, the authors alone are responsible for any remaining errors and for the views expressed herein.

** Department of Economics, Washington University, Campus Box 1208, 1 Brookings Drive, St. Louis, MO 63130-4899 Phone: (1-314) 935-8486, Fax: (1-314) 935-4156, e-mail: berliant@artsci.wustl.edu

${ }^{ \pm}$Institute of Economic Research, Kyoto University, Yoshida-Honmachi, Sakyo-ku, Kyoto, 606-01 Japan Phone: (81-75) 753-7122, Fax (81-75) 753-7198, e-mail: fujita@kier.kyotou.ac.jp 


\section{Introduction}

\subsection{The Research Agenda}

In this paper we examine the microdynamics of knowledge creation and transfer by using a simple model. With a focus on the two person case, the basic principles of this complex process can be uncovered. Although the two person model admittedly has its limitations, particularly in the dynamic choice of partners for knowledge creation and transfer, it has its advantages in analytical tractability because we can solve the equilibrium dynamics explicitly. ${ }^{1}$

Our major research questions are as follows. How do knowledge creation and transfer occur? How do they perpetuate themselves? How do agents change during this process? Are the equilibrium dynamics efficient?

As people create and transfer knowledge, they change. Thus, the history of meetings and their content is important. If two people meet for a long time, then their base of knowledge in common increases, and their partnership eventually becomes less productive. Similarly, if two persons have very different knowledge bases, they have little common ground for communication, so their partnership will not be very productive.

For these reasons, we attempt to model endogenous agent heterogeneity, or horizontal agent differentiation, to look at the permanent effects of knowledge creation and growth. Thus, we are examining how social capital is accumulated at a micro level. Our model is analytically tractable, so we do not have to resort to simulations; we find each equilibrium path explicitly. The model is also at an intermediate level of aggregation. That is, although it is at a more micro level than large aggregate models such as those found in the endogenous growth literature, we do not work out completely its microfoundations. That is left to future research.

The analogy between partner dancing and working jointly to create and exchange knowledge is useful, so we will use terms from these activities interchangeably. Knowledge creation, exchange, and individual production all occur simultaneously at each point in time. The dancers can work alone or with their partner. They work together if and only if they both agree that it is useful. Production always occurs at a rate proportional to the agent's current stock of knowledge, as does knowledge creation when an agent dances alone.

\footnotetext{
${ }^{1}$ Elsewhere, in Berliant and Fujita (2006), we provide extensions of the model and results to the context of the general case of any number of potential partners, but to maintain analytical tractability, we do not consider asymmetric states or knowledge transfer.
} 
The suitability of their dance partner depends on the stock of knowledge they have in common and their respective stocks of exclusive knowledge. The fastest rate of knowledge creation occurs when these factors are in balance.

Our results are summarized as follows. First, in a two person model where myopic agents can decide whether or not to work with each other, there exist many sink points in the interaction game, depending discontinuously on initial heterogeneity. The most interesting of these features too much homogeneity relative to the most productive state.

For simplicity, we employ a deterministic framework. It seems possible to add stochastic elements to the model, but at the cost of complexity. It should also be possible to employ the law of large numbers to a more basic stochastic framework to obtain equivalent results.

Next we compare our work to the balance of the literature. Section 2 gives the model and notation, Section 3 analyzes equilibrium in the case of two participants or dancers, Section 4 examines welfare in the two person model, whereas Section 5 provides our conclusions and suggestions for future dancing. Two appendices provide the proofs of key results.

The basic framework that employs knowledge creation as a black box driving economic growth is usually called the endogenous growth model. Here we make a modest attempt to open that black box. The literature using this black box includes Shell (1966), Romer (1986, 1990), Lucas (1988), Jones and Manuelli (1990), and many papers building on these contributions. There are two key features of our model in relation to the endogenous growth literature. First, our agents are heterogeneous, and that heterogeneity is endogenous to the model. Second, the effectiveness of the externality between agents working together can change over time, and this change is endogenous.

Fujita and Weber (2003) consider a model where heterogeneity between agents is exogenous and discrete. They examine the effects of immigration policy on the productivity and welfare of workers. They note that progress in technology in a country where workers are highly trained is in small steps involving intensive interactions between workers and a relatively homogeneous work force, whereas countries that specialize in production of new knowledge have a relatively heterogeneous work force. This motivates our examination of how endogenous worker heterogeneity affects industrial structure, the speed of innovation, and the pattern of worker interaction.

Differentiation of agents in terms of quality (or vertical characteristics) of knowledge is studied in Jovanovic and Rob (1989) in the context of a search 
model. In contrast, our model examines (endogenous) horizontal heterogeneity of agents and its effect on knowledge creation, knowledge transfer, and consumption.

\section{The Model - Ideas and Knowledge}

In this section, we introduce the basic concepts of our model of ideas and knowledge.

An idea is represented by a box. It has a label on it that everyone can read (the label is common knowledge in the game we shall describe). This label describes the contents. Each box contains an idea that is described by its label. Learning the actual contents of the box, as opposed to its label, takes time, so although anyone can read the label on the box, they cannot understand its contents without investing time. This time is used to open the box and to understand fully its contents. An example is a recipe for making "udon noodles as in Takamatsu." It is labelled as such, but would take time to learn. Another example is reading a paper in a journal. Its label or title can be understood quickly, but learning the contents of the paper requires an investment of time. Production of a new paper, which is like opening a new box, either jointly or individually, also takes time.

Suppose we have an infinite number of boxes, each containing a different piece of knowledge, which is what we call an idea. We put them in a row in an arbitrary order.

There are 2 persons, $i$ and $j$, in the economy. We assume that each person has a replica of the infinite row of boxes introduced above, and that each copy of the row has the same order. Our model features continuous time. Fix time $t \in \mathbb{R}_{+}$and consider person $i$. A box is indexed by $k=1,2, \ldots$ Take any box $k$. If person $i$ knows the idea inside that box, we put a sticker on it that says 1 ; otherwise, we put a sticker on it that says 0 . That is, let $x_{i}^{k}(t) \in\{0,1\}$ be the sticker on box $k$ for person $i$ at time $t$. The state of knowledge, or just knowledge, of person $i$ at time $t$ is thus defined to be $K_{i}(t)=\left(x_{i}^{1}(t), x_{i}^{2}(t), \ldots\right) \in\{0,1\}^{\infty}$. The reason we use an infinite vector of possible ideas is that we are using an infinite time horizon, and there are always new ideas that might be discovered, even in the preparation of udon noodles. Given $K_{i}(t)$ with only finitely many non-zero components, there is an infinite number of ideas that could be created in the next step.

In this paper, we will treat ideas symmetrically. All that will matter is the 
number an agent knows at a particular time. Extensions to idea hierarchies and knowledge structures will be discussed in the conclusions.

Given $K_{i}(t)=\left(x_{i}^{1}(t), x_{i}^{2}(t), \ldots\right)$,

$$
n_{i}(t)=\sum_{k=1}^{\infty} x_{i}^{k}(t)
$$

represents the number of ideas known by person $i$ at time $t$. Next, we will define the number of ideas that two persons, $i$ and $j$, both know. Assume that $j \neq i$. Define $K_{j}(t)=\left(x_{j}^{1}(t), x_{j}^{2}(t), \ldots\right)$ and

$$
n_{i j}^{c}(t)=\sum_{k=1}^{\infty} x_{i}^{k}(t) \cdot x_{j}^{k}(t)
$$

So $n_{i j}^{c}(t)$ represents the number of ideas known by both persons $i$ and $j$ at time $t$. Notice that $i$ and $j$ are symmetric in this definition, so $n_{i j}^{c}(t)=n_{j i}^{c}(t)$. Define

$$
n_{i j}^{d}(t)=n_{i}(t)-n_{i j}^{c}(t)
$$

to be the number of ideas known by person $i$ but not known by person $j$ at time $t$.

Knowledge is a set of ideas that are possessed by a person at a particular time. However, knowledge is not a static concept. New knowledge can be produced either individually or jointly, and ideas can be shared with others. But all of this activity takes time.

Next we describe the components of the rest of the model. Consider first a model with just two agents, $i$ and $j$. At each time, each faces a decision about whether or not to meet with the other. If both want to meet at a particular time, a meeting will occur. If either does not want to meet, then they do not meet. If the agents do not meet at a given time, then they produce separately and also create new knowledge separately. If the two persons do decide to meet at a given time, then they share older knowledge together and create new knowledge together.

So consider a given time $t$. In order to explain how knowledge creation, knowledge exchange, and commodity production work, it is useful for intuition (but not technically necessary) to view this time period of fixed length as consisting of subperiods of fixed length. Each individual is endowed with a fixed amount of labor that is supplied inelastically during the period. In the first subperiod, individual production takes place. We shall assume constant returns to scale in physical production, so it is not beneficial for individuals 
to collaborate in production. Each individual uses their labor during the first subperiod to produce consumption good on their own, whether or not they are meeting. We shall assume below that although there are no increasing returns to scale in production, the productivity of a person's labor depends on their stock of knowledge. Activity in the second subperiod depends on whether or not there is a meeting. If there is no meeting, then each person spends the second subperiod creating new knowledge on their own. Evidently, the new knowledge created during this subperiod can differ between the two persons, because they are not communicating. They open different boxes. Since there is an infinity of different boxes, the probability that the two agents will open the same box (even at different points in time), either working by themselves or in distinct meetings, is assumed to be zero. If there is a meeting, then the second subperiod is divided into two parts. In the first part, the two persons who are meeting spend their time (and labor) sharing old knowledge, boxes they have opened in previous time periods that the other person has not opened. In the second part, they create new knowledge together, so they open boxes together. We wish to emphasize that the division of a time period into subperiods is purely an expositional device. Rigorously, whether or not a meeting occurs determines how much attention is devoted to the various activities at a given time.

What do the agents know when they face the decision about whether or not to meet at time $t$ ? Each person knows both $K_{i}(t)$ and $K_{j}(t)$. In other words, each person is aware of their own knowledge and is also aware of the other's knowledge. Thus, they also know $n_{i}(t), n_{j}(t), n_{i j}^{c}(t)=n_{j i}^{c}(t), n_{i j}^{d}(t)$, and $n_{j i}^{d}(t)$ when they decide whether or not to meet at time $t$. The notation for whether or not a meeting actually occurs at time $t$ is: $\delta_{i j}(t) \equiv \delta_{j i}(t)=1$ if a meeting occurs and $\delta_{i j}(t) \equiv \delta_{j i}(t)=0$ if no meeting occurs at time $t$. Meetings only occur if both persons agree that a meeting should take place.

Next, we must specify the dynamics of the knowledge system and the objectives of the people in the model in order to determine whether or not they decide to meet at a particular time. In order to accomplish this, it is easiest to abstract away from the notation for specific boxes, $K_{i}(t)$, and to focus on the dynamics of the quantity statistics related to knowledge, $n_{i}(t), n_{j}(t)$, $n_{i j}^{c}(t)=n_{j i}^{c}(t), n_{i j}^{d}(t)$, and $n_{j i}^{d}(t)$. Since we are treating ideas symmetrically, in a sense these quantities are sufficient statistics for our analysis.

The simplest piece of the model to specify is what happens if there is no meeting and the two people thus work in isolation. Let $a_{i}(t)$ be the rate of 
creation of new ideas created by person $i$ and let $a_{j}(t)$ be the rate of creation of new ideas created by $j$, both at time $t$. Let $b_{i j}(t)$ and $b_{j i}(t)$ be the rate of transfer of ideas from $i$ to $j$ and from $j$ to $i$, respectively, at time $t^{2}$ Then we assume that the creation of new knowledge during isolation $\left(\delta_{i j}(t)=0\right)$ is governed by the following equations:

$$
\begin{aligned}
a_{i}(t) & =\alpha \cdot n_{i}(t) \text { and } a_{j}(t)=\alpha \cdot n_{j}(t) \text { when } \delta_{i j}(t)=0 . \\
b_{i j}(t) & =0 \text { and } b_{j i}(t)=0 \text { when } \delta_{i j}(t)=0 .
\end{aligned}
$$

So we assume that if there is no meeting at time $t$, individual knowledge grows at a rate proportional to the knowledge already acquired by an individual. Meanwhile, knowledge held commonly by the two persons does not grow. In particular, ideas are not shared.

If a meeting does occur at time $t\left(\delta_{i j}(t)=1\right)$, then both knowledge exchange between the two persons and joint knowledge creation occur. When a meeting takes place, joint knowledge creation is governed by the following dynamics $:^{3}$

$$
a_{i j}(t)=\beta \cdot\left[n_{i j}^{c}(t) \cdot n_{i j}^{d}(t) \cdot n_{j i}^{d}(t)\right]^{\frac{1}{3}}
$$

So when the two people meet, joint knowledge creation occurs at a rate proportional to the normalized product of their knowledge in common, the individual knowledge of $i$, and the individual knowledge of $j$. The rate of creation of new knowledge is highest when the proportions of ideas in common, ideas exclusive to person $i$, and ideas exclusive to person $j$ are split evenly. Ideas in common are necessary for communication, while ideas exclusive to one person or the other imply more heterogeneity or originality in the collaboration. If one person in the collaboration does not have exclusive ideas, there is no reason for the other person to meet and collaborate. The multiplicative nature of the function in equation (5) drives the relationship between knowledge creation and the relative proportions of ideas in common and ideas exclusive to one or the other agent.

\footnotetext{
${ }^{2}$ In principle, all of these time-dependent quantities are positive integers. However, for simplicity we take them to be continuous (in $\mathbb{R}_{+}$) throughout the paper. One interpretation is that the creation or sharing of an idea occurs at a stochastic time, and the real numbers are taken to be the probability of a jump in a Poisson process. The justification of the use of a real number instead of an integer seems to add little but complication to the analysis.

${ }^{3}$ We may generalize equation (5) as follows:

$$
a_{i j}(t)=\max \left\{(\alpha-\varepsilon) n_{i}(t),(\alpha-\varepsilon) n_{j}(t), \beta\left[n_{i j}^{c}(t) \cdot n_{i j}^{d}(t) \cdot n_{j i}^{d}(t)\right]^{\frac{1}{3}}\right\}
$$

where $\varepsilon>0$ represents the costs from the lack of concentration. This generalization, however, does not change the results presented in this paper in any essential way.
} 
Under these circumstances, no knowledge creation in isolation occurs. During meetings at time $t$, knowledge transfer occurs in addition to the creation of new knowledge. Knowledge transfer is governed by the following dynamics:

$$
\begin{aligned}
b_{i j}(t) & =\gamma \cdot\left[n_{i j}^{d}(t) \cdot n_{i j}^{c}(t)\right]^{\frac{1}{2}} \\
b_{j i}(t) & =\gamma \cdot\left[n_{j i}^{d}(t) \cdot n_{i j}^{c}(t)\right]^{\frac{1}{2}}
\end{aligned}
$$

So when a meeting occurs, knowledge transfer from $i$ to $j$ happens at a rate proportional to the normalized product of the number of ideas that person $i$ has but that person $j$ does not have, and the ideas common to both persons. The explanation is that communication is necessary for knowledge transfer, so the two persons must have some ideas in common $\left(n_{i j}^{c}(t)\right)$. But in addition, person $i$ must have some ideas that are not already possessed by person $j$ $\left(n_{i j}^{d}(t)\right)$. The same intuition applies to knowledge transfer in the opposite direction from $j$ to $i$, represented by the second equation in (6). The change in the number of ideas that both persons have in common $\left(\dot{n}_{i j}^{c}(t)\right)$ is the sum of knowledge transfers in both directions and the new ideas jointly created. From person $i$ 's perspective, the number of ideas that $i$ has but $j$ doesn't have $\left(n_{i j}^{d}(t)\right)$ decreases with knowledge transfers from $i$ to $j$. Finally, the change in the number of ideas possessed by person $i$ is the sum of the ideas that are jointly created and the number of ideas transferred from $j$ to $i$. The analogous statements hold for the variables associated with $j$.

Let us focus on agent $i$ (the equations for agent $j$ are analogous). With a meeting, we have the following dynamics incorporating both knowledge creation and transfer:

$$
\begin{aligned}
\dot{n}_{i}(t) & =a_{i j}(t)+b_{j i}(t) \\
\dot{n}_{i j}^{c}(t) & =a_{i j}(t)+b_{i j}(t)+b_{j i}(t) \\
\dot{n}_{i j}^{d}(t) & =-b_{i j}(t)
\end{aligned}
$$

Given this structure, we can define the rates of idea innovation and knowledge transfer at time $t$, depending on whether or not a meeting occurs.

$$
\begin{aligned}
\dot{n}_{i}(t)= & {\left[1-\delta_{i j}(t)\right] \cdot \alpha \cdot n_{i}(t)+} \\
& \delta_{i j}(t) \cdot\left(\beta \cdot\left[n_{i j}^{c}(t) \cdot n_{i j}^{d}(t) \cdot n_{j i}^{d}(t)\right]^{\frac{1}{3}}+\gamma \cdot\left[n_{j i}^{d}(t) \cdot n_{i j}^{c}(t)\right]^{\frac{1}{2}}\right) \\
\dot{n}_{i j}^{c}(t)= & \delta_{i j}(t) \cdot\left(\beta \cdot\left[n_{i j}^{c}(t) \cdot n_{i j}^{d}(t) \cdot n_{j i}^{d}(t)\right]^{\frac{1}{3}}+\gamma \cdot\left[n_{j i}^{d}(t) \cdot n_{i j}^{c}(t)\right]^{\frac{1}{2}}\right. \\
& \left.+\gamma \cdot\left[n_{i j}^{d}(t) \cdot n_{j i}^{c}(t)\right]^{\frac{1}{2}}\right) \\
\dot{n}_{i j}^{d}(t)= & {\left[1-\delta_{i j}(t)\right] \cdot \alpha \cdot n_{i}(t)-\delta_{i j}(t) \cdot \gamma \cdot\left[n_{i j}^{d}(t) \cdot n_{j i}^{c}(t)\right]^{\frac{1}{2}} }
\end{aligned}
$$


Whether a meeting occurs or not, there is production in each period for both persons. Felicity in that time period is defined to be the quantity of output. ${ }^{4}$ Define $y_{i}(t)$ to be production output (or felicity) for person $i$ at time $t$, and define $y_{j}(t)$ to be production output (or felicity) of person $j$ at time $t$. Normalizing the coefficient of production to be 1 , we take

$$
y_{i}(t)=n_{i}(t)
$$

so

$$
\dot{y}_{i}(t)=\dot{n}_{i}(t)
$$

By definition,

$$
\frac{\dot{y}_{i}(t)}{y_{i}(t)}=\frac{\dot{n}_{i}(t)}{n_{i}(t)}
$$

which represents the rate of growth of income.

Finally, we must define the rule used by each person to decide whether they want a meeting at time $t$ or not. Formally,

$$
\begin{gathered}
\delta_{i j}(t)=1 \Longleftrightarrow \\
\beta \cdot\left[n_{i j}^{c}(t) \cdot n_{i j}^{d}(t) \cdot n_{j i}^{d}(t)\right]^{\frac{1}{3}}+\gamma \cdot\left[n_{j i}^{d}(t) \cdot n_{i j}^{c}(t)\right]^{\frac{1}{2}}>\alpha \cdot n_{i}(t) \text { and } \\
\beta \cdot\left[n_{j i}^{c}(t) \cdot n_{j i}^{d}(t) \cdot n_{i j}^{d}(t)\right]^{\frac{1}{3}}+\gamma \cdot\left[n_{i j}^{d}(t) \cdot n_{j i}^{c}(t)\right]^{\frac{1}{2}}>\alpha \cdot n_{j}(t)
\end{gathered}
$$

To keep the model tractable in this first analysis, we assume a myopic rule. So a person would like a meeting if and only if the increase in their rate of output with a meeting is higher than the increase in their rate of output without a meeting. ${ }^{5}$ Note that we use the increase in the rate of output rather than the rate of output since in a continuous time model, the rate of output at time $t$ is unaffected by the decision about whether to meet made at time $t$.

This completes the statement of the model. Dropping the time dependence of variables to analyze dynamics, we obtain the following equations of motion.

$$
\begin{aligned}
\dot{y}_{i}= & \dot{n}_{i}=\left[1-\delta_{i j}\right] \cdot \alpha \cdot n_{i}+ \\
& \delta_{i j} \cdot\left(\beta \cdot\left[n_{i j}^{c} \cdot n_{i j}^{d} \cdot n_{j i}^{d}\right]^{\frac{1}{3}}+\gamma \cdot\left[n_{j i}^{d} \cdot n_{i j}^{c}\right]^{\frac{1}{2}}\right) \\
\dot{n}_{i j}^{c}= & \delta_{i j} \cdot\left(\beta \cdot\left[n_{i j}^{c} \cdot n_{i j}^{d} \cdot n_{j i}^{d}\right]^{\frac{1}{3}}+\gamma \cdot\left[n_{j i}^{d} \cdot n_{i j}^{c}\right]^{\frac{1}{2}}+\gamma \cdot\left[n_{i j}^{d} \cdot n_{j i}^{c}\right]^{\frac{1}{2}}\right) \\
\dot{n}_{i j}^{d}= & {\left[1-\delta_{i j}\right] \cdot \alpha \cdot n_{i}-\delta_{i j} \cdot \gamma \cdot\left[n_{i j}^{d} \cdot n_{j i}^{c}\right]^{\frac{1}{2}} }
\end{aligned}
$$

This system, with analogous equations for agent $j$, represents a partner dance.

\footnotetext{
${ }^{4}$ Given that the focus of this paper is on knowledge creation and transfer rather than production, we use the simplest possible form for the production function.

${ }^{5}$ We will see that the rule used in the case of ties is not important.
} 


\section{Equilibrium Dynamics}

In order to analyze our system, we first divide all of our equations by the total number of ideas possessed by $i$ and $j$ :

$$
n^{i j}=n_{i j}^{d}+n_{j i}^{d}+n_{i j}^{c}
$$

and define new variables

$$
\begin{aligned}
& m_{i j}^{c} \equiv m_{j i}^{c}=\frac{n_{i j}^{c}}{n^{i j}}=\frac{n_{j i}^{c}}{n^{i j}} \\
& m_{i j}^{d}=\frac{n_{i j}^{d}}{n^{i j}}, m_{j i}^{d}=\frac{n_{j i}^{d}}{n^{i j}}
\end{aligned}
$$

From (11), we obtain

$$
1=m_{i j}^{d}+m_{j i}^{d}+m_{i j}^{c}
$$

After some detailed calculations (see Appendix a of the technical appendix for all of the steps), we obtain $\dot{m}_{i j}^{d}$ and $\dot{m}_{j i}^{d}$ as functions of $m_{i j}^{d}$ and $m_{j i}^{d}$ only, as follows.

$$
\begin{aligned}
\dot{m}_{i j}^{d}= & {\left[1-\delta_{i j}\right] \cdot \alpha \cdot\left\{\left(1-m_{i j}^{d}\right)\left(1-m_{i j}^{d}-m_{j i}^{d}\right)\right\} } \\
& -\delta_{i j} \cdot\left\{\gamma \cdot\left[m_{i j}^{d} \cdot\left(1-m_{i j}^{d}-m_{j i}^{d}\right)\right]^{\frac{1}{2}}+m_{i j}^{d} \cdot \beta \cdot\left[\left(1-m_{i j}^{d}-m_{j i}^{d}\right) \cdot m_{i j}^{d} \cdot m_{j i}^{d}\right]^{\frac{1}{3}}\right\} \\
\dot{m}_{j i}^{d}= & {\left[1-\delta_{i j}\right] \cdot \alpha \cdot\left\{\left(1-m_{j i}^{d}\right)\left(1-m_{i j}^{d}-m_{j i}^{d}\right)\right\} } \\
& -\delta_{i j} \cdot\left\{\gamma \cdot\left[m_{j i}^{d} \cdot\left(1-m_{i j}^{d}-m_{j i}^{d}\right)\right]^{\frac{1}{2}}+m_{j i}^{d} \cdot \beta \cdot\left[\left(1-m_{i j}^{d}-m_{j i}^{d}\right) \cdot m_{j i}^{d} \cdot m_{i j}^{d}\right]^{\frac{1}{3}}\right\}
\end{aligned}
$$

To study this more, we must study (9) further. Deleting time indices and dividing by $n^{i j}$,

$$
\begin{aligned}
& \delta_{i j}=1 \Longleftrightarrow \\
& \beta \cdot\left[m_{i j}^{c} \cdot m_{i j}^{d} \cdot m_{j i}^{d}\right]^{\frac{1}{3}}+\gamma \cdot\left[m_{j i}^{d} \cdot m_{i j}^{c}\right]^{\frac{1}{2}}>\alpha \cdot\left(1-m_{j i}^{d}\right) \\
& \text { and } \beta \cdot\left[m_{i j}^{c} \cdot m_{j i}^{d} \cdot m_{i j}^{d}\right]^{\frac{1}{3}}+\gamma \cdot\left[m_{i j}^{d} \cdot m_{i j}^{c}\right]^{\frac{1}{2}}>\alpha \cdot\left(1-m_{i j}^{d}\right)
\end{aligned}
$$

Substituting further,

$$
\begin{aligned}
& \delta_{i j}=1 \Longleftrightarrow \\
& \beta \cdot\left[\left(1-m_{j i}^{d}-m_{i j}^{d}\right) \cdot m_{i j}^{d} \cdot m_{j i}^{d}\right]^{\frac{1}{3}}+\gamma \cdot\left[m_{j i}^{d} \cdot\left(1-m_{j i}^{d}-m_{i j}^{d}\right)\right]^{\frac{1}{2}}>\alpha \cdot\left(1-m_{j i}^{d}\right) \\
& \text { and } \beta \cdot\left[\left(1-m_{j i}^{d}-m_{i j}^{d}\right) \cdot m_{j i}^{d} \cdot m_{i j}^{d}\right]^{\frac{1}{3}}+\gamma \cdot\left[m_{i j}^{d} \cdot\left(1-m_{j i}^{d}-m_{i j}^{d}\right)\right]^{\frac{1}{2}}>\alpha \cdot\left(1-m_{i j}^{d}\right)
\end{aligned}
$$


In other words, meetings occur when the rate of growth of income or utility of each person is higher with a meeting than without a meeting.

Define

$$
\begin{aligned}
F_{i}\left(m_{i j}^{d}, m_{j i}^{d}\right)= & \beta \cdot\left[\left(1-m_{j i}^{d}-m_{i j}^{d}\right) \cdot m_{i j}^{d} \cdot m_{j i}^{d}\right]^{\frac{1}{3}}+ \\
& \gamma \cdot\left[m_{j i}^{d} \cdot\left(1-m_{j i}^{d}-m_{i j}^{d}\right)\right]^{\frac{1}{2}}-\alpha \cdot\left(1-m_{j i}^{d}\right) \\
F_{j}\left(m_{i j}^{d}, m_{j i}^{d}\right)= & \beta \cdot\left[\left(1-m_{j i}^{d}-m_{i j}^{d}\right) \cdot m_{j i}^{d} \cdot m_{i j}^{d}\right]^{\frac{1}{3}}+ \\
& \gamma \cdot\left[m_{i j}^{d} \cdot\left(1-m_{j i}^{d}-m_{i j}^{d}\right)\right]^{\frac{1}{2}}-\alpha \cdot\left(1-m_{i j}^{d}\right)
\end{aligned}
$$

and

$$
\begin{aligned}
& M_{i}=\left\{\left(m_{i j}^{d}, m_{j i}^{d}\right) \in \mathbb{R}_{+}^{2} \mid m_{i j}^{d}+m_{j i}^{d} \leq 1, F_{i}\left(m_{i j}^{d}, m_{j i}^{d}\right)>0\right\} \\
& M_{j}=\left\{\left(m_{i j}^{d}, m_{j i}^{d}\right) \in \mathbb{R}_{+}^{2} \mid m_{i j}^{d}+m_{j i}^{d} \leq 1, F_{j}\left(m_{i j}^{d}, m_{j i}^{d}\right)>0\right\}
\end{aligned}
$$

whereas

$$
M=M_{i} \cap M_{j}
$$

The function $F_{i}\left(m_{i j}^{d}, m_{j i}^{d}\right)$ represents the net benefit for $i$ of meeting instead of isolation. Likewise for $F_{j}\left(m_{i j}^{d}, m_{j i}^{d}\right)$. The set $M_{i}$ represents those pairs $\left(m_{i j}^{d}, m_{j i}^{d}\right)$ such that $i$ wants to meet with $j$, since for these pairs, the rate of growth of $i$ 's utility or income with a meeting is higher than the rate of growth of $i$ 's utility or income without a meeting. The set $M_{j}$ represents those pairs $\left(m_{i j}^{d}, m_{j i}^{d}\right)$ such that $j$ wants to meet with $i$. Of course, the set $M$ represents those pairs $\left(m_{i j}^{d}, m_{j i}^{d}\right)$ such that both persons want to meet with each other. Thus, meetings will occur at time $t$ for pairs in $M$.

We represent our model in our Figures as a function of $m_{i j}^{d}$ and $m_{j i}^{d}$; since $m_{i j}^{d}+m_{j i}^{d}+m_{i j}^{c}=1$, we know that $1-m_{i j}^{c}=m_{i j}^{d}+m_{j i}^{d} \leq 1$, where this inequality is represented by half of the unit square (a triangle) in $\mathbb{R}^{2}$. We put $m_{i j}^{d}$ on the horizontal axis and $m_{j i}^{d}$ on the vertical axis, omitting $m^{c}$.

Figure 1, panels (a) and (b) illustrate the sets $M_{i}$ and $M_{j}$, respectively, for $\beta=\gamma=1$ and for various values of $\alpha$. Of course, panels (a) and (b) are mirror images of each other across the $45^{\circ}$ line. Figure 2 illustrates $M$, the set of pairs where both persons want to meet, and its complement, where no meetings occur, for the same parameter values. When $\left(m_{i j}^{d}, m_{j i}^{d}\right)$ is close to the boundary of the triangle, meetings do not occur. The reason is that the two persons have too little in common to interact effectively (near the downward sloping diagonal) or someone has too little exclusive knowledge (near the axes) to interact effectively. Meetings only take place in the interior where the three components of knowledge are relatively balanced.

\section{FIGURES 1 AND 2 GO HERE}


In fact we can describe the properties of the set $M$ in general. The set $M$ has the shape depicted in Figure 2; see Appendix b of the technical appendix for proof. In particular, $M$ is roughly the shape of an apple core aligned on the $45^{\circ}$ line. As $\alpha$ increases, the productivity of creating ideas alone increases, so people are less likely to want to meet to create, implying that each $M_{i}$ and $M_{j}$ shrinks as $\alpha$ increases, as does $M$. If $\alpha$ is a little more than $1, M$ disappears. To be precise, let $M(\alpha)$ be the set $M$ under the parameter value $\alpha$. Then, whenever $\alpha_{1}<\alpha_{2}$, the set $M\left(\alpha_{2}\right)$ is entirely contained in $M\left(\alpha_{1}\right)$. Thus, as shown in Figure 2, there is a unique point $B$ contained in every $M(\alpha)$, provided $M(\alpha)$ is nonempty. We call $B$ the bliss point, for the point $B$ in Figure 2 is the point where the rate of increase in income or utility is maximized for each person, as we will explain in the next section (see also Lemma A6 in Appendix c of the technical appendix).

Next we discuss the dynamics of the system. Consider first the case where there is no meeting, so $\delta_{i j}=0$ is fixed exogenously. Then from equations (13), the dynamics are given by the following equations:

$$
\begin{aligned}
& \dot{m}_{i j}^{d}=\alpha \cdot\left(1-m_{i j}^{d}\right)\left(1-m_{i j}^{d}-m_{j i}^{d}\right) \\
& \dot{m}_{j i}^{d}=\alpha \cdot\left(1-m_{j i}^{d}\right)\left(1-m_{i j}^{d}-m_{j i}^{d}\right)
\end{aligned}
$$

\section{FIGURE 3 GOES HERE}

Figure 3, panel (a) illustrates the gradient field assuming that $\delta_{i j}=0$. Several facts follow quickly from these derivations. First, if there is no meeting $\left(\delta_{i j}=0\right)$, then both $\dot{m}_{i j}^{d}$ and $\dot{m}_{j i}^{d}$ are non-negative, and positive on the interior of the triangle. So if there is no meeting, the vector field points to the northeast. Furthermore, in the lower half of the triangle where $m_{i j}^{d} \geq m_{j i}^{d}$ (the other part is symmetric), we have

$$
\frac{\dot{m}_{j i}^{d}}{\dot{m}_{i j}^{d}}=\frac{1-m_{j i}^{d}}{1-m_{i j}^{d}} \geq 1
$$

where the inequality is strict off of the diagonal. Thus, when $\delta_{i j}=0$, the vector field points northeast but toward the diagonal. Under the assumption of no meeting, the system tends to sink points along the diagonal line where $m_{i j}^{d}+m_{j i}^{d}=1$, illustrated in Figure 3, panel (a) by a line between $(0,1)$ and $(1,0)$.

Figure 3, panel (b) illustrates the gradient field assuming that $\delta_{i j}=1$. Then (13) implies: 


$$
\begin{aligned}
& \dot{m}_{i j}^{d}=-\gamma \cdot\left[m_{i j}^{d} \cdot\left(1-m_{i j}^{d}-m_{j i}^{d}\right)\right]^{\frac{1}{2}}+m_{i j}^{d} \cdot \beta \cdot\left[\left(1-m_{i j}^{d}-m_{j i}^{d}\right) \cdot m_{i j}^{d} \cdot m_{j i}^{d}\right]^{\frac{1}{3}} \\
& \dot{m}_{j i}^{d}=-\gamma \cdot\left[m_{j i}^{d} \cdot\left(1-m_{i j}^{d}-m_{j i}^{d}\right)\right]^{\frac{1}{2}}+m_{j i}^{d} \cdot \beta \cdot\left[\left(1-m_{i j}^{d}-m_{j i}^{d}\right) \cdot m_{j i}^{d} \cdot m_{i j}^{d}\right]^{\frac{1}{3}}
\end{aligned}
$$

Both of these expressions are negative on the interior of the triangle and the vector field points southwest. Consider, for convenience, the lower half of the triangle where $m_{i j}^{d} \geq m_{j i}^{d}$; the other part is symmetric. Then

$$
\begin{aligned}
\frac{\dot{m}_{j i}^{d}}{\dot{m}_{i j}^{d}} & =\frac{\gamma \cdot\left[m_{j i}^{d} \cdot\left(1-m_{i j}^{d}-m_{j i}^{d}\right)\right]^{\frac{1}{2}}+m_{j i}^{d} \cdot \beta \cdot\left[\left(1-m_{i j}^{d}-m_{j i}^{d}\right) \cdot m_{j i}^{d} \cdot m_{i j}^{d}\right]^{\frac{1}{3}}}{\gamma \cdot\left[m_{i j}^{d} \cdot\left(1-m_{i j}^{d}-m_{j i}^{d}\right)\right]^{\frac{1}{2}}+m_{i j}^{d} \cdot \beta \cdot\left[\left(1-m_{i j}^{d}-m_{j i}^{d}\right) \cdot m_{i j}^{d} \cdot m_{j i}^{d}\right]^{\frac{1}{3}}} \\
& \leq 1
\end{aligned}
$$

where the inequality is strict off of the diagonal. Thus, the vector field points southwest but toward the diagonal, as illustrated in Figure 3, panel (b). The only sink is at $(0,0)$, so the system eventually moves there under the assumption of a meeting.

Next, we combine the case where there is no meeting $\left(\delta_{i j}=0\right)$ with the case where there is a meeting $\left(\delta_{i j}=1\right)$, and let the agents choose whether or not to meet. This is illustrated in Figure 4.

\section{FIGURE 4 GOES HERE}

The model follows the dynamics for meetings $\left(\delta_{i j}=1\right)$ on $M$ and the dynamics for no meetings $\left(\delta_{i j}=0\right)$ on the complement of $M$.

In general, there is a continuum of stable points of the system, corresponding to the points where $m_{i j}^{d}+m_{j i}^{d}=1$. For these points, eventually the myopic return to no meeting dominates the returns to meetings, since eventually the two persons have almost nothing in common. These stable points, however, are not very interesting.

We have not completely specified the dynamics. This is especially important on the boundary of $M$, where at least one person is indifferent between meeting and not meeting. We take an arbitrarily small unit of time, $\Delta t$, and assume that if at least one person becomes indifferent between meeting and not meeting, but the two persons are currently meeting, then the meeting must continue for at least $\Delta t$ units of time. Similarly, if the two persons are not meeting when one person becomes indifferent between meeting and not meeting while the other wants to meet or is indifferent, then they cannot meet for at least $\Delta t$ units of time. So if a person becomes indifferent between meeting 
or not meeting at time $t$, the function $\delta_{i j}(t)$ cannot change its value until time $t+\Delta t$. Finally, when at least one person initially happens to be on the boundary of $M$ (that is, at least one person is indifferent between meeting and not meeting), then they cannot meet for at least $\Delta t$ units of time. Under this set of rules, we can be more specific about the dynamic process near the boundary of $M$.

In terms of dynamics, if the system does not evolve toward the uninteresting stable points where there are no meetings (and the two people have nothing in common), eventually the system reaches the southwest boundary of the set $M$. From there, the assumption that $\delta_{i j}$ is constant over time intervals of at least length $\Delta t$ at the boundary of $M$ will drive the system in a zigzag process toward the place furthest to the southwest and on the diagonal that is a member of $M$. In other words, this is the point $J=\left(m^{d}, m^{d}\right) \in M$ with lowest norm. It is the remaining stable point of our model. Small movements around $J$ will continue due to our assumption about the dynamics at the boundary of $M$, namely that meetings or isolation are sticky. As $\Delta t \rightarrow 0$, the process converges to the point $J$. The point $J$ features symmetry between the two agents with a large degree of homogeneity relative to the remainder of the points in $M$ and the other points in the triangle generally.

So given various initial compositions of knowledge $\left(m_{i j}^{d}, m_{j i}^{d}\right)$, where will the system end up? If the initial composition of knowledge is relatively unbalanced, in other words near the boundary of the triangle, the sink will be a point on the diagonal where $m_{i j}^{d}+m_{j i}^{d}=1$. If the initial composition of knowledge is relatively balanced, then the sink will be the point $J$.

Using the facts about the shape of $M$, the point $J$ exists and is unique as long as $M \neq \emptyset$.

At the point $J=\left(m^{J}, m^{J}\right), m^{J} \leq \frac{2}{5}$, for reasons explained in the next section.

Without loss of generality, we can allow $\delta_{i j}$ to take values in $[0,1]$ rather than $\{0,1\}$. The interpretation of a fractional $\delta_{i j}$ is that at each instant of time, a person divides their time between a meeting $\delta_{i j}$ proportion of that instant and isolation $\left(1-\delta_{i j}\right)$ proportion of that instant. All of our results concerning the model when $\delta_{i j}$ is restricted to $\{0,1\}$ carry over to the case where $\delta_{i j} \in[0,1]$. The reason is that except on the boundary of $M$, persons strictly prefer $\delta_{i j} \in\{0,1\}$ to fractional values of $\delta_{i j}$, as each person's objective function is linear in $\delta_{i j}$. On the boundary of $M$, our rule concerning dynamics prevents $\delta_{i j}$ from taking on fractional values, as it must retain its value from 
the previous iteration of the process for at least time $\Delta t>0$. So if the process pierces the boundary from inside $M$, it must retain $\delta_{i j}=1$ for an additional time of at least $\Delta t$. If it pierces the boundary from outside $M$, it must retain $\delta_{i j}=0$ for an additional time of at least $\Delta t$. It may seem trivial to allow fractional $\delta_{i j}$ when discussing equilibrium behavior, but allowing fractional $\delta_{i j}$ is crucial to the next section, where we consider efficiency.

\section{Efficiency}

To construct an analog of Pareto efficiency in this model, we use a social planner who can choose whether or not people should meet in each time period. As noted above, we shall allow the social planner to choose values of $\delta_{i j}$ in $[0,1]$, so that persons can be required to meet for a percentage of the total time in a period, and not meet for the remainder of the period. To avoid dependence of our notion of efficiency on a discount rate, we employ the following alternative concepts. The first is stronger than the second. A path of $\delta_{i j}$ is a piecewise continuous function of time (on $[0, \infty)$ ) taking values in $[0,1]$. For each path of $\delta_{i j}$, there corresponds a unique time path of $m_{i j}^{d}$ determined by equation (13), respecting the initial condition, and thus a unique time path of income $y_{i}\left(t ; \delta_{i j}\right)$. We say that a path $\delta_{i j}^{\prime}$ (strictly) dominates a path $\delta_{i j}$ if

$$
y_{i}\left(t ; \delta_{i j}^{\prime}\right) \geq y_{i}\left(t ; \delta_{i j}\right) \text { and } y_{j}\left(t ; \delta_{i j}^{\prime}\right) \geq y_{j}\left(t ; \delta_{i j}\right) \text { for all } t \geq 0
$$

with strict inequality for at least one over a positive interval of time. As this concept is quite strong, and thus difficult to use as an efficiency criterion, it will sometimes be necessary to employ a weaker concept, which we discuss next. We say that a path $\delta_{i j}$ is overtaken by a path $\delta_{i j}^{\prime}$ if there exists a $t^{\prime}$ such that

$$
y_{i}\left(t ; \delta_{i j}^{\prime}\right) \geq y_{i}\left(t ; \delta_{i j}\right) \text { and } y_{j}\left(t ; \delta_{i j}^{\prime}\right) \geq y_{j}\left(t ; \delta_{i j}\right) \text { for all } t>t^{\prime}
$$

with strict inequality for at least one over a positive interval of time.

Two types of sink points were analyzed in the last section. First consider equilibrium paths that have $m^{J}$ as the sink point; they reach $m^{J}$ in finite time and stay there. Using Figure 5, we will construct an alternative path $\delta_{i j}^{\prime}$ that dominates the equilibrium path $\delta_{i j}$.

\section{FIGURE 5 GOES HERE}


In constructing this path, we will make use of income changes along the upward sloping diagonal in Figure 4. Setting

$$
\begin{aligned}
m_{i j}^{d} & =m_{j i}^{d}=m \\
y_{i} & =y_{j}=y
\end{aligned}
$$

we use (10) and (11) to obtain

$$
\begin{aligned}
\frac{\dot{y}(t)}{y(t)}= & \frac{\dot{y}(t)}{n_{i}(t)}=\frac{\dot{y}(t)}{n(t)[1-m(t)]} \\
= & {\left[1-\delta_{i j}(t)\right] \cdot \alpha+\delta_{i j}(t) \cdot\left\{\beta \cdot\left[\left(1-\frac{m(t)}{1-m(t)}\right) \cdot\left(\frac{m(t)}{1-m(t)}\right)^{2}\right]^{\frac{1}{3}}\right.} \\
& \left.\left.+\gamma \cdot\left[\left(1-\frac{m(t)}{1-m(t)}\right) \cdot\left(\frac{m(t)}{1-m(t)}\right)\right]^{\frac{1}{2}}\right)\right\}
\end{aligned}
$$

To simplify notation, we define the growth rate when the two persons meet, $\delta_{i j}=1$, as

$$
\begin{aligned}
g(m)= & \beta \cdot\left[\left(1-\frac{m}{1-m}\right) \cdot\left(\frac{m}{1-m}\right)^{2}\right]^{\frac{1}{3}} \\
& \left.+\gamma \cdot\left[\left(1-\frac{m}{1-m}\right) \cdot\left(\frac{m}{1-m}\right)\right]^{\frac{1}{2}}\right)
\end{aligned}
$$

Thus

$$
\frac{\dot{y}(t)}{y(t)}=\left[1-\delta_{i j}\right] \cdot \alpha+\delta_{i j} \cdot g(m)
$$

Figure 5 illustrates the graph of the function $g(m)$ as a bold line for $\beta=$ $\gamma=1$. We can show ${ }^{6}$ that $g(m)$ is strictly quasi-concave on $\left[0, \frac{1}{2}\right]$, achieving its maximal value at $m^{B} \in\left[\frac{1}{3}, \frac{2}{5}\right]$. We can also show (see Lemma A6 of the technical appendix) that $m=m^{B}$ corresponds to the bliss point $B$ in Figure 2. In other words, whenever $M \neq \emptyset, B=\left(m^{B}, m^{B}\right) \in M$, so the point $J=\left(m^{J}, m^{J}\right)$ defined in Figure 4 and in the previous section has the property that $m^{J} \leq 2 / 5$, as it is defined to be the point in $M$ on the diagonal and closest to the origin. We define the point $I=\left(m^{I}, m^{I}\right)$ in Figure 4 to be the point in $M$ on the diagonal and farthest from the origin.

Let $t^{\prime}$ be the time at which the equilibrium path reaches $\left(m^{J}, m^{J}\right)$. Let the planner set $\delta_{i j}^{\prime}(t)=\delta_{i j}(t)$ for $t \leq t^{\prime}$, taking the same path as the equilibrium path until $t^{\prime}$. From this time on, the planner uses only symmetric points, namely those on the upward sloping diagonal in Figure 4; these points comprise the horizontal axis in Figure 5. At time $t^{\prime}$, the planner takes $\delta_{i j}^{\prime}(t)=0$ until

\footnotetext{
${ }^{6}$ See Lemma A6 of the technical appendix.
} 
$\left(m^{I}, m^{I}\right)$ is attained, prohibiting meetings so that the dancers can profit from ideas created in isolation. Then the planner sets $\delta_{i j}^{\prime}(t)=1$ until $\left(m^{J}, m^{J}\right)$ is attained, permitting meetings and the development of more knowledge in common. The last two phases are repeated as necessary.

From Figure 5 , the income paths $y_{i}\left(t ; \delta_{i j}^{\prime}\right)$ and $y_{j}\left(t ; \delta_{i j}^{\prime}\right)$ generated by the path $\delta_{i j}^{\prime}$ clearly dominate the income paths $y_{i}\left(t ; \delta_{i j}\right)$ and $y_{j}\left(t ; \delta_{i j}\right)$ generated by the equilibrium path $\delta_{i j}$. Thus, the equilibrium is far from the most productive path.

Next consider equilibrium paths $\delta_{i j}(t)$ that end in sink points on the downward sloping diagonal in Figure 4. Our dominance criterion cannot be used in this situation, since in potentially dominating plans, the planner will need to force the couple to meet outside of region $M$ in Figure 4 in early time periods. During this time interval, the dancers could do better by not meeting, and thus a comparison of the income derived from the paths would rely on the discount rate, something we are trying to avoid. So we will use our weaker criterion here, that of overtaking.

Given an equilibrium path $\delta_{i j}(t)$ with sink point on the diagonal, the planner can construct an overtaking path $\delta_{i j}^{\prime}(t)$ as follows. The first phase is to construct a path $\delta_{i j}^{\prime}(t)$ that reaches a point in region $M$ in finite time. Such a path can readily be constructed using Figures 3 and $4 .^{7}$ After reaching region $M$, the second and third phases are the same as described above for the construction of a path that dominates one ending with $m^{J}$. Since the paths with sinks on the downward sloping diagonal have income growth $\alpha$ at every time, whereas the new path $\delta_{i j}^{\prime}(t)$ features income growth that exceeds $\alpha$ whenever the couple is meeting, $\delta_{i j}^{\prime}(t)$ overtakes $\delta_{i j}(t)$.

The most productive state $m^{B}$ is characterized by less homogeneity than the stable point $m^{J}$. Of course, attaining $m^{B}$ requires the social planner to force the two persons not to meet some of the time. Otherwise, the system evolves toward more homogeneity.

\footnotetext{
${ }^{7}$ Such a path can be constructed as follows. In Figure 2 or Figure 4, take the union of all closed, one dimensional intervals parallel to the $45^{\circ}$ line with one endpoint on an axis and the other endpoint a member of $M$. Call this set $M^{\prime}$. From time 0 , take $\delta=1$. Using Figure 3(b), the path hits $M^{\prime}$ in finite time. From this time on, take $\delta=0$. Using Figure $3(\mathrm{a})$, the path hits $M$ in finite time.
} 


\section{Conjectures and Conclusions}

We have considered a model of knowledge creation and exchange that is based on individual behavior, allowing myopic agents to decide whether joint or individual production is best for them at any given time. This is a pure externality model of knowledge creation, with no markets.

In the present context of two people, there are a continuum of sink points (equilibria) for the knowledge accumulation process. Every state where the two agents have a negligible proportion of ideas in common is attainable as an equilibrium for some initial condition. There is one additional and more interesting sink, involving a large degree of homogeneity as well as symmetry of the two agents, and this is attainable from a non-negligible set of initial conditions. Relative to the efficient state, the first set of sink points has agents that are too heterogeneous, while the second sink point has agents that are too homogeneous. ${ }^{8}$

Of course, the major limitation of this work is the use of only two people. In other work, we employ more agents, but at a cost, namely the absence of knowledge transfer while limiting ourselves to symmetric states.

Here we discuss the many alternate directions for future work and extensions of the framework. Chief among these are the introduction of foresight on the part of agents, the introduction of stochastic elements into the model, and the introduction of side payments. Though the two person model is limited, extensions of the basic framework are much easier. It is apparent from the analysis in section 3 that limited foresight, in the form of short sightedness instead of perfect myopia, will not be enough to overturn our results. In order to completely overturn the results of section 2 , the agents must have long range foresight. In this case, they can construct more efficient paths as in section 3. Moreover, long range foresight in combination with side payments could produce tutelage when the initial state is asymmetric. When the person with more knowledge is willing to accept payment for teaching, the equilibrium paths can look very different from what we have proposed.

In the international context, where each country is often represented by one agent, our model might be applicable. Two countries or two regions would be assigned representative agents. In that context, knowledge creation and transfer, especially as related to developed and developing countries, would be of interest. Analogs of the transfer paradox would be quite fascinating. ${ }^{9}$

\footnotetext{
${ }^{8}$ The proximate cause is agent myopia.

${ }^{9}$ We refer to our companion paper, Berliant and Fujita (2006), for further discussion of
} 


\section{References}

[1] Berliant, M., Fujita, M., 2006. Knowledge Creation as a Square Dance on the Hilbert Cube. Mimeo. http://econpapers.repec.org/paper/wpawuwpga/0506006.htm

[2] Fujita, M. and S. Weber, 2003. Strategic immigration policies and welfare in heterogeneous countries. Discussion Paper No. 569, Kyoto Institute of Economic Research, Kyoto University.

[3] Jones, L., Manuelli, R., 1990. A convex model of equilibrium growth: Theory and policy implications. Journal of Political Economy 98, 1008-1038.

[4] Jovanovic, B. and R. Rob, 1989. The growth and diffusion of knowledge. The Review of Economic Studies 56, 569-582.

[5] Lucas, R. E., Jr., 1988. On the mechanics of economic development. Journal of Monetary Economics 22, 2-42.

[6] Romer, P., 1986. Increasing returns and long-run growth. Journal of Political Economy 94, 1002-1037.

[7] Romer, P., 1990. Endogenous technological change. Journal of Political Economy 98, S71-S102.

[8] Shell, K., 1966. Toward a theory of inventive activity and capital accumulation. American Economic Review 61, 62-68.

extensions. 
(a)

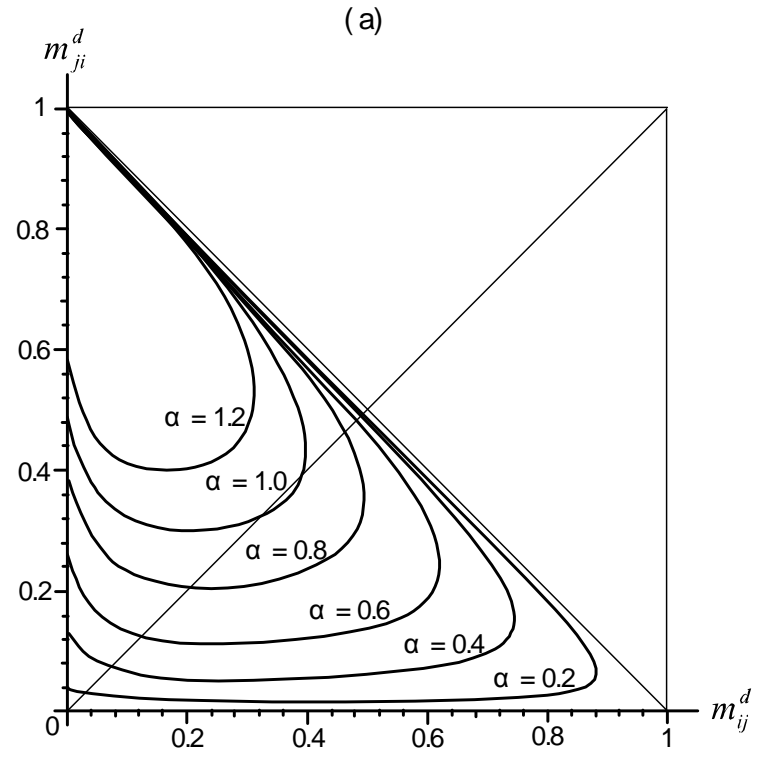

(b)

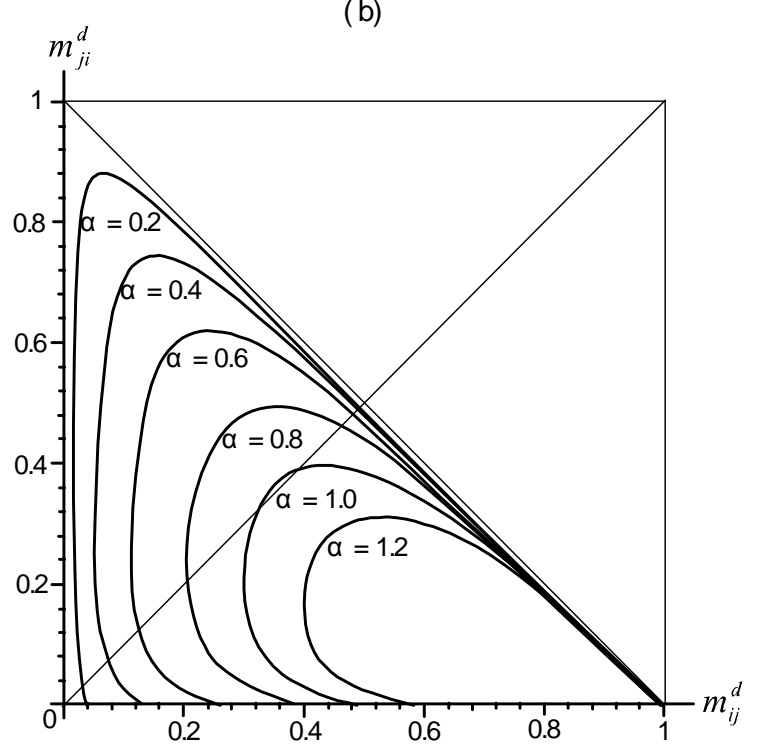

Figure 1: The sets $M_{i}$ and $M_{j}$ under various values of $\alpha$.

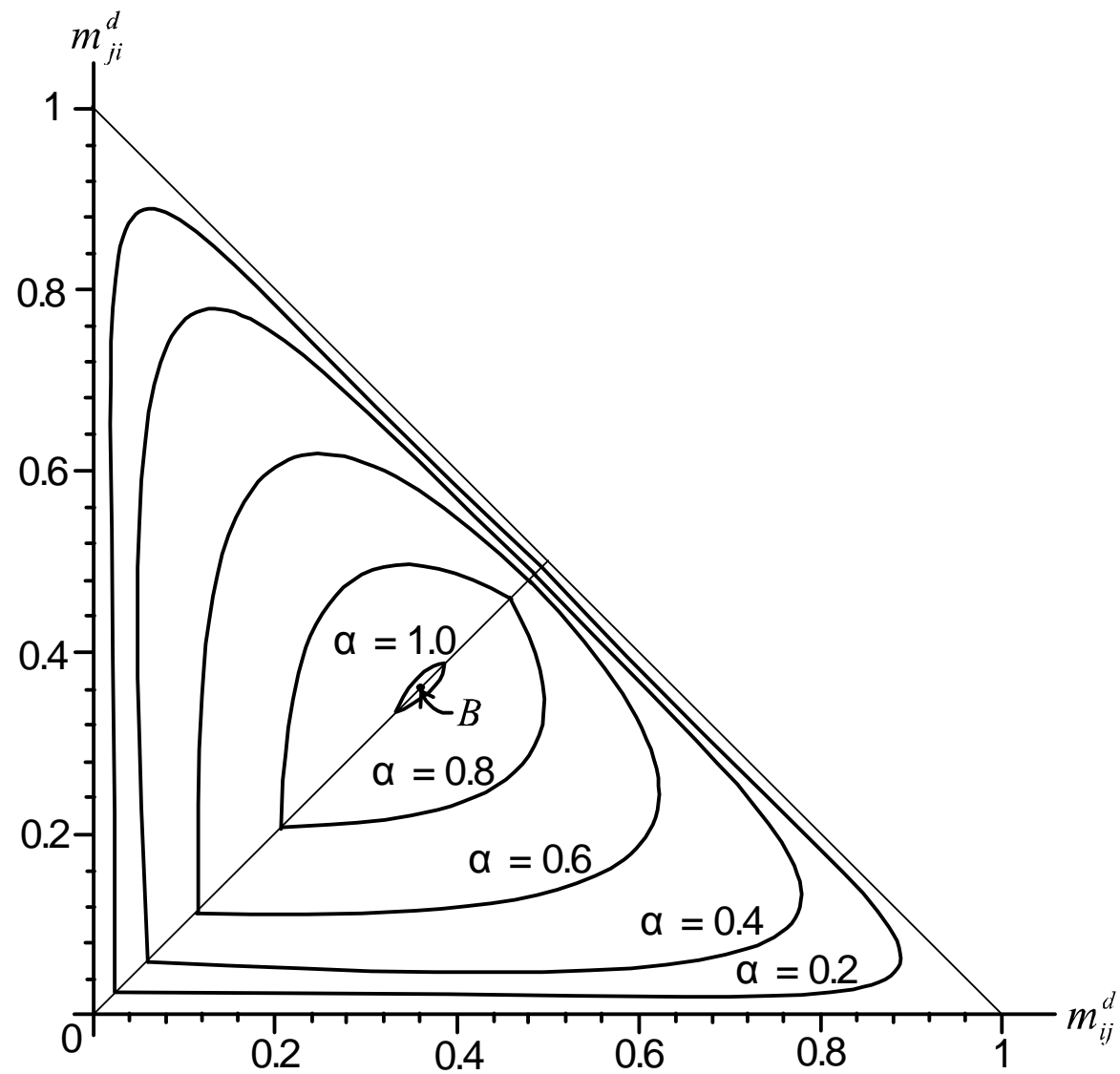

Figure 2: The set $M$ under various values of $\alpha$, and the bliss point $B$. 

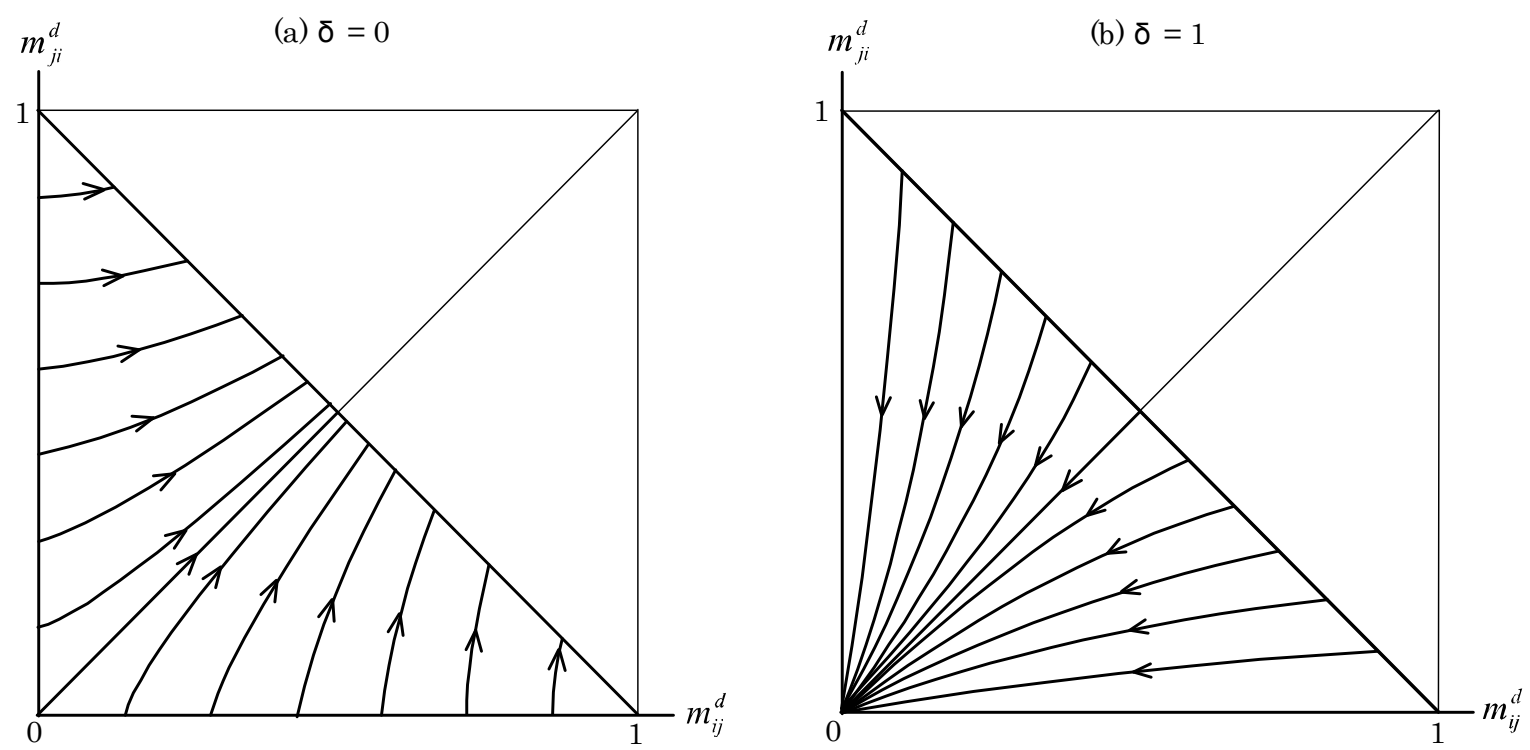

Figure 3: Dynamics under a fixed value of $\delta$.

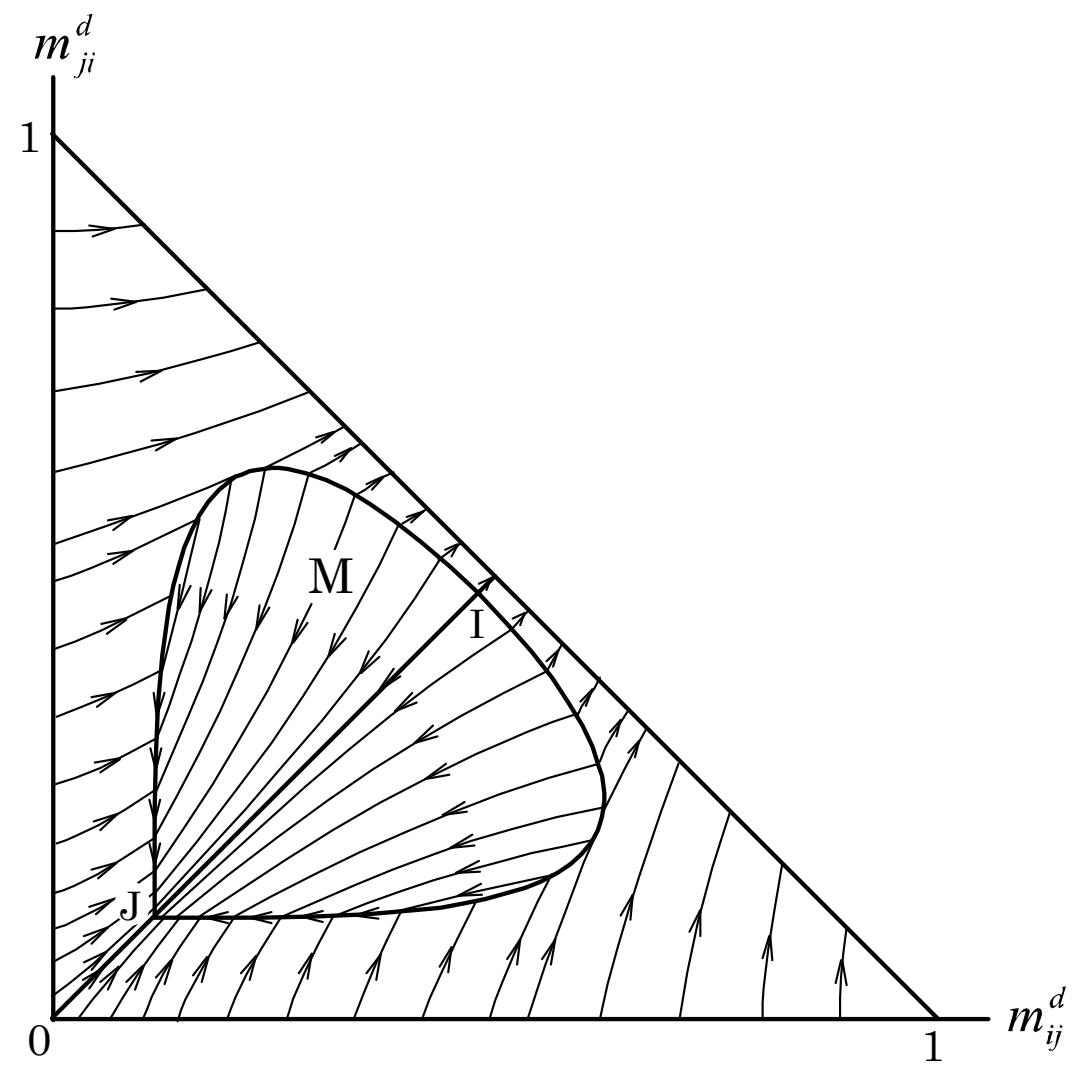

Figure 4: Dynamics with $\delta$ endogenous. 


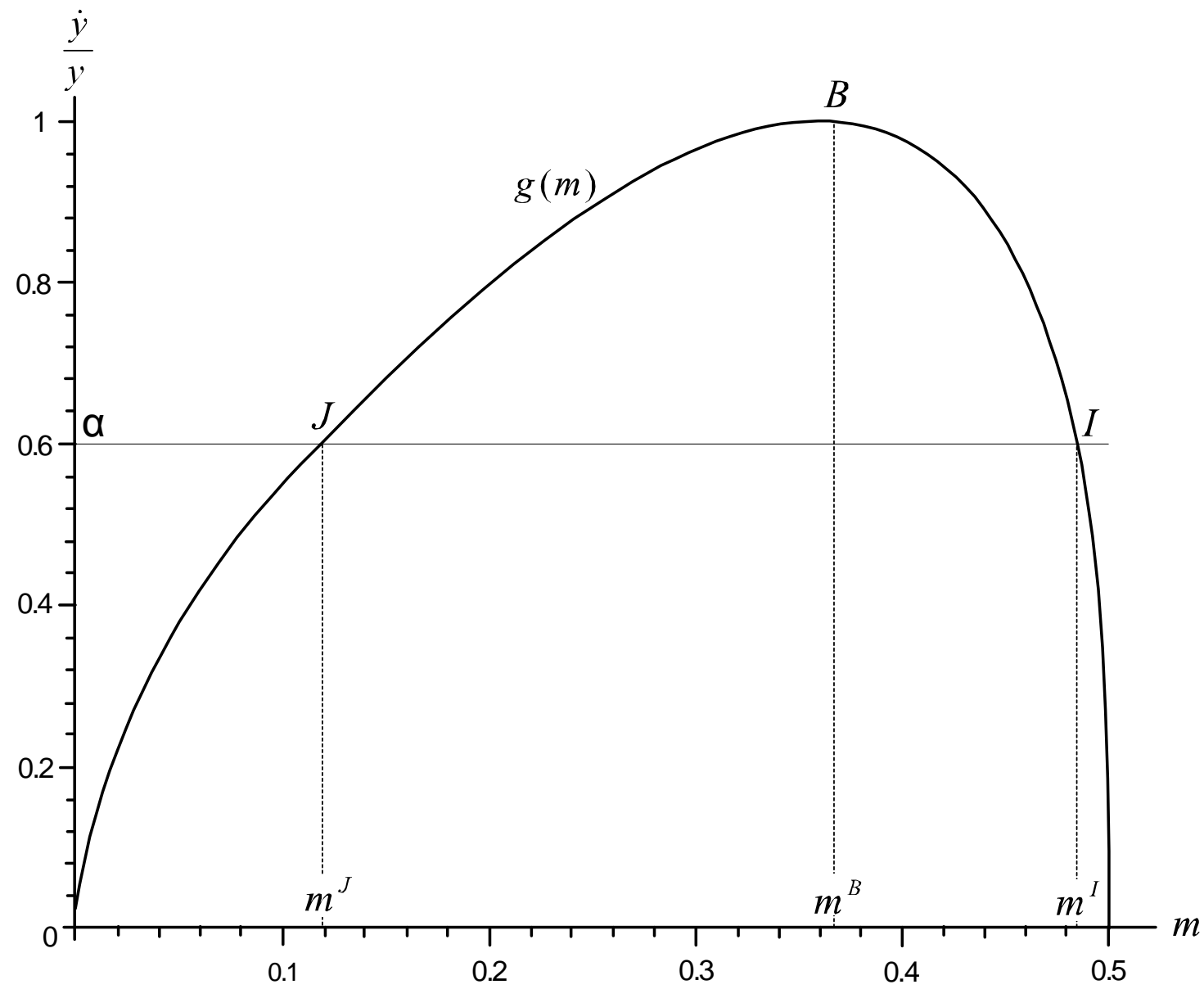

Figure 5: Efficiency and the bliss point

\section{Technical Appendix}

\subsection{Appendix a}

Theorem A1: Knowledge dynamics evolve according to the system:

$$
\begin{aligned}
\dot{m}_{i j}^{d}= & {\left[1-\delta_{i j}\right] \cdot \alpha \cdot\left\{\left(1-m_{i j}^{d}\right)\left(1-m_{i j}^{d}-m_{j i}^{d}\right)\right\} } \\
& -\delta_{i j} \cdot\left\{\gamma \cdot\left[m_{i j}^{d} \cdot\left(1-m_{i j}^{d}-m_{j i}^{d}\right)\right]^{\frac{1}{2}}+m_{i j}^{d} \cdot \beta \cdot\left[\left(1-m_{i j}^{d}-m_{j i}^{d}\right) \cdot m_{i j}^{d} \cdot m_{j i}^{d}\right]^{\frac{1}{3}}\right\} \\
\dot{m}_{j i}^{d}= & {\left[1-\delta_{j i}\right] \cdot \alpha \cdot\left\{\left(1-m_{j i}^{d}\right)\left(1-m_{i j}^{d}-m_{j i}^{d}\right)\right\} } \\
& -\delta_{j i} \cdot\left\{\gamma \cdot\left[m_{j i}^{d} \cdot\left(1-m_{i j}^{d}-m_{j i}^{d}\right)\right]^{\frac{1}{2}}+m_{j i}^{d} \cdot \beta \cdot\left[\left(1-m_{i j}^{d}-m_{j i}^{d}\right) \cdot m_{j i}^{d} \cdot m_{i j}^{d}\right]^{\frac{1}{3}}\right\}
\end{aligned}
$$

Proof of Theorem A1: Recalling (3),

$$
m_{i j}^{d}+m^{c}=1-m_{j i}^{d}
$$


and dividing by $n^{i j}$ yields

$$
\begin{aligned}
\frac{\dot{y}_{i}}{n^{i j}}= & \frac{\dot{n}_{i}}{n^{i j}}=\left[1-\delta_{i j}\right] \cdot \alpha \cdot\left(1-m_{j i}^{d}\right)+ \\
& \delta_{i j} \cdot\left(\beta \cdot\left[m^{c} \cdot m_{i j}^{d} \cdot m_{j i}^{d}\right]^{\frac{1}{3}}+\gamma \cdot\left[m_{j i}^{d} \cdot m^{c}\right]^{\frac{1}{2}}\right) \\
\frac{\dot{n}_{i j}^{c}}{n^{i j}}= & \delta_{i j} \cdot\left(\beta \cdot\left[m^{c} \cdot m_{i j}^{d} \cdot m_{j i}^{d}\right]^{\frac{1}{3}}+\gamma \cdot\left[m_{j i}^{d} \cdot m^{c}\right]^{\frac{1}{2}}\right. \\
& \left.+\gamma \cdot\left[m_{i j}^{d} \cdot m^{c}\right]^{\frac{1}{2}}\right) \\
\frac{\dot{n}_{i j}^{d}}{n^{i j}=} & {\left[1-\delta_{i j}\right] \cdot \alpha \cdot\left(1-m_{j i}^{d}\right)-\delta_{i j} \cdot \gamma \cdot\left[m_{i j}^{d} \cdot m^{c}\right]^{\frac{1}{2}} }
\end{aligned}
$$

Substituting (12) for $m^{c}$,

$$
\begin{aligned}
\frac{\dot{y}_{i}}{n^{i j}=} & \frac{\dot{n}_{i}}{n^{i j}}=\left[1-\delta_{i j}\right] \cdot \alpha \cdot\left(1-m_{j i}^{d}\right)+ \\
& \delta_{i j} \cdot\left(\beta \cdot\left[\left(1-m_{i j}^{d}-m_{j i}^{d}\right) \cdot m_{i j}^{d} \cdot m_{j i}^{d}\right]^{\frac{1}{3}}+\gamma \cdot\left[m_{j i}^{d} \cdot\left(1-m_{i j}^{d}-m_{j i}^{d}\right)\right]^{\frac{1}{2}}\right) \\
\frac{\dot{n}_{i j}^{c}}{n^{i j}=} & \delta_{i j} \cdot\left(\beta \cdot\left[\left(1-m_{i j}^{d}-m_{j i}^{d}\right) \cdot m_{i j}^{d} \cdot m_{j i}^{d}\right]^{\frac{1}{3}}+\gamma \cdot\left[m_{j i}^{d} \cdot\left(1-m_{i j}^{d}-m_{j i}^{d}\right)\right]^{\frac{1}{2}}\right. \\
& \left.+\gamma \cdot\left[m_{i j}^{d} \cdot\left(1-m_{i j}^{d}-m_{j i}^{d}\right)\right]^{\frac{1}{2}}\right) \\
\frac{\dot{n}_{i j}^{d}=}{n^{i j}=} & {\left[1-\delta_{i j}\right] \cdot \alpha \cdot\left(1-m_{j i}^{d}\right)-\delta_{i j} \cdot \gamma \cdot\left[m_{i j}^{d} \cdot\left(1-m_{i j}^{d}-m_{j i}^{d}\right)\right]^{\frac{1}{2}} }
\end{aligned}
$$


Now

$$
\begin{aligned}
\dot{m}_{i j}^{d}= & \frac{d\left(n_{i j}^{d} / n^{i j}\right)}{d t} \\
= & \frac{\dot{n}_{i j}^{d}}{n^{i j}}-\frac{n_{i j}^{d} \cdot \dot{n}^{i j}}{\left(n^{i j}\right)^{2}} \\
= & \frac{\dot{n}_{i j}^{d}}{n^{i j}}-\frac{n_{i j}^{d}}{n^{i j}} \cdot \frac{\dot{n}^{i j}}{n^{i j}} \\
= & {\left[1-\delta_{i j}\right] \cdot \alpha \cdot\left(1-m_{j i}^{d}\right)-\delta_{i j} \cdot \gamma \cdot\left[m_{i j}^{d} \cdot\left(1-m_{i j}^{d}-m_{j i}^{d}\right)\right]^{\frac{1}{2}}-m_{i j}^{d} \cdot\left(\frac{\dot{n}_{i j}^{d}}{n}+\frac{\dot{n}_{j i}^{d}}{n}+\frac{\dot{n}_{i j}^{c}}{n}\right) } \\
= & {\left[1-\delta_{i j}\right] \cdot \alpha \cdot\left(1-m_{j i}^{d}\right)-\delta_{i j} \cdot \gamma \cdot\left[m_{i j}^{d} \cdot\left(1-m_{i j}^{d}-m_{j i}^{d}\right)\right]^{\frac{1}{2}} } \\
& -m_{i j}^{d} \cdot\left\{\left[1-\delta_{i j}\right] \cdot \alpha \cdot\left(1-m_{j i}^{d}\right)-\delta_{i j} \cdot \gamma \cdot\left[m_{i j}^{d} \cdot\left(1-m_{i j}^{d}-m_{j i}^{d}\right)\right]^{\frac{1}{2}}+\left[1-\delta_{i j}\right] \cdot \alpha \cdot\left(1-m_{i j}^{d}\right.\right. \\
& -\delta_{i j} \cdot \gamma \cdot\left[m_{j i}^{d} \cdot\left(1-m_{i j}^{d}-m_{j i}^{d}\right)\right]^{\frac{1}{2}}+\delta_{i j} \cdot\left(\beta \cdot\left[\left(1-m_{i j}^{d}-m_{j i}^{d}\right) \cdot m_{i j}^{d} \cdot m_{j i}^{d}\right]^{\frac{1}{3}}\right. \\
& \left.\left.+\gamma \cdot\left[m_{j i}^{d} \cdot\left(1-m_{i j}^{d}-m_{j i}^{d}\right)\right]^{\frac{1}{2}}+\gamma \cdot\left[m_{i j}^{d} \cdot\left(1-m_{i j}^{d}-m_{j i}^{d}\right)\right]^{\frac{1}{2}}\right)\right\} \\
= & {\left[1-\delta_{i j}\right] \cdot \alpha \cdot\left(1-m_{j i}^{d}\right)-\delta_{i j} \cdot \gamma \cdot\left[m_{i j}^{d} \cdot\left(1-m_{i j}^{d}-m_{j i}^{d}\right)\right]^{\frac{1}{2}} } \\
& -m_{i j}^{d} \cdot\left\{\left[1-\delta_{i j}\right] \cdot \alpha \cdot\left(1-m_{j i}^{d}\right)+\left[1-\delta_{i j}\right] \cdot \alpha \cdot\left(1-m_{i j}^{d}\right)\right. \\
& \left.+\delta_{i j} \cdot \beta \cdot\left[\left(1-m_{i j}^{d}-m_{j i}^{d}\right) \cdot m_{i j}^{d} \cdot m_{j i}^{d}\right]^{\frac{1}{3}}\right\} \\
= & {\left[1-\delta_{i j}\right] \cdot \alpha \cdot\left\{1-m_{j i}^{d}-2 m_{i j}^{d}+\left(m_{i j}^{d}\right)^{2}+m_{i j}^{d} \cdot m_{j i}^{d}\right\} } \\
& -\delta_{i j} \cdot\left\{\gamma \cdot\left[m_{i j}^{d} \cdot\left(1-m_{i j}^{d}-m_{j i}^{d}\right)\right]^{\frac{1}{2}}+m_{i j}^{d} \cdot \beta \cdot\left[\left(1-m_{i j}^{d}-m_{j i}^{d}\right) \cdot m_{i j}^{d} \cdot m_{j i}^{d}\right]^{\frac{1}{3}}\right\} \\
= & {\left[1-\delta_{i j}\right] \cdot \alpha \cdot\left\{\left(1-m_{i j}^{d}\right)\left(1-m_{j i}^{d}\right)-m_{i j}^{d}+\left(m_{i j}^{d}\right)^{2}\right\} } \\
& -\delta_{i j} \cdot\left\{\gamma \cdot\left[m_{i j}^{d} \cdot\left(1-m_{i j}^{d}-m_{j i}^{d}\right)\right]^{\frac{1}{2}}+m_{i j}^{d} \cdot \beta \cdot\left[\left(1-m_{i j}^{d}-m_{j i}^{d}\right) \cdot m_{i j}^{d} \cdot m_{j i}^{d}\right]^{\frac{1}{3}}\right\} \\
= & {\left[1-\delta_{i j}\right] \cdot \alpha \cdot\left\{\left(1-m_{i j}^{d}\right)\left(1-m_{j i}^{d}\right)-m_{i j}^{d}\left(1-m_{i j}^{d}\right)\right\} } \\
& -\delta_{i j} \cdot\left\{\gamma \cdot\left[m_{i j}^{d} \cdot\left(1-m_{i j}^{d}-m_{j i}^{d}\right)\right]^{\frac{1}{2}}+m_{i j}^{d} \cdot \beta \cdot\left[\left(1-m_{i j}^{d}-m_{j i}^{d}\right) \cdot m_{i j}^{d} \cdot m_{j i}^{d}\right]^{\frac{1}{3}}\right\} \\
= & {\left[1-\delta_{i j}\right] \cdot \alpha \cdot\left\{\left(1-m_{i j}^{d}\right)\left(1-m_{j i}^{d}-m_{i j}^{d}\right)\right\} } \\
& -\delta_{i j} \cdot\left\{\gamma \cdot\left[m_{i j}^{d} \cdot\left(1-m_{i j}^{d}-m_{j i}^{d}\right)\right]^{\frac{1}{2}}+m_{i j}^{d} \cdot \beta \cdot\left[\left(1-m_{i j}^{d}-m_{j i}^{d}\right) \cdot m_{i j}^{d} \cdot m_{j i}^{d}\right]^{\frac{1}{3}}\right\} \\
& \\
n & \\
n &
\end{aligned}
$$

The fourth line follows from (11), that implies

$$
\frac{\dot{n}^{i j}}{n^{i j}}=\frac{\dot{n}_{i j}^{d}}{n^{i j}}+\frac{\dot{n}_{j i}^{d}}{n^{i j}}+\frac{\dot{n}_{i j}^{c}}{n^{i j}}
$$

Symmetric calculations hold for $\dot{m}_{j i}^{d}$.

\subsection{Appendix $b$}

Theorem A2: Suppose that $\left(m_{i j}^{d}, m_{j i}^{d}\right) \in M$. Then $\left(m_{j i}^{d}, m_{i j}^{d}\right) \in M$ and the line segment $\left[\left(m_{i j}^{d}, m_{j i}^{d}\right),\left(m_{j i}^{d}, m_{i j}^{d}\right)\right] \subseteq M$. In particular, if $M \neq \emptyset$, then it contains a point on the diagonal segment $[(0,0),(1,1)]$. Moreover, the diagonal intersected with $M$ is a convex set. In fact, every line parallel to the diagonal 
intersected with $M$ is a convex set. Finally, every point in $M \cap((0,0),(1,1))$ has a neighborhood contained in $M$.

To prove Theorem A2, we proceed with a sequence of lemmata. First we need some definitions to make notation easier.

\section{Definitions:}

$$
\begin{aligned}
& f\left(m, m^{\prime}\right)=\beta \cdot\left[\left(1-m-m^{\prime}\right) \cdot m \cdot m^{\prime}\right]^{\frac{1}{3}} \\
& h\left(m, m^{\prime}\right)=\gamma \cdot\left[\left(1-m-m^{\prime}\right) \cdot m^{\prime}\right]^{\frac{1}{2}}
\end{aligned}
$$

With these definitions, the equations defining $M_{i}(15)$ and $M_{j}(16)$ become:

$$
\begin{aligned}
& f\left(m_{i j}^{d}, m_{j i}^{d}\right)+h\left(m_{i j}^{d}, m_{j i}^{d}\right)-\alpha \cdot\left(1-m_{j i}^{d}\right)>0 \\
& f\left(m_{j i}^{d}, m_{i j}^{d}\right)+h\left(m_{j i}^{d}, m_{i j}^{d}\right)-\alpha \cdot\left(1-m_{i j}^{d}\right)>0
\end{aligned}
$$

Lemma A1: $\left(m_{i j}^{d}, m_{j i}^{d}\right) \in M_{i}$ and $m_{i j}^{d} \geq m_{j i}^{d}$ imply $\left(m_{j i}^{d}, m_{i j}^{d}\right) \in M_{i}$. $\left(m_{i j}^{d}, m_{j i}^{d}\right) \in M_{j}$ and $m_{i j}^{d} \leq m_{j i}^{d}$ imply $\left(m_{j i}^{d}, m_{i j}^{d}\right) \in M_{j}$.

Proof of Lemma A1: $f\left(m_{i j}^{d}, m_{j i}^{d}\right)=f\left(m_{j i}^{d}, m_{i j}^{d}\right) . \frac{h\left(m_{j i}^{d}, m_{i j}^{d}\right)}{h\left(m_{i j}^{d}, m_{j i}^{d}\right)}=\left[\frac{m_{i j}^{d}}{m_{j i}^{d}}\right]^{\frac{1}{2}} \geq 1$, since $m_{i j}^{d} \geq m_{j i}^{d} . \quad\left(m_{i j}^{d}, m_{j i}^{d}\right) \in M_{i}$ implies $f\left(m_{i j}^{d}, m_{j i}^{d}\right)+h\left(m_{i j}^{d}, m_{j i}^{d}\right)-\alpha(1-$ $\left.m_{j i}^{d}\right)>0$. Since $h\left(m_{j i}^{d}, m_{i j}^{d}\right) \geq h\left(m_{i j}^{d}, m_{j i}^{d}\right)$ and $m_{i j}^{d} \geq m_{j i}^{d}, f\left(m_{j i}^{d}, m_{i j}^{d}\right)+$ $h\left(m_{j i}^{d}, m_{i j}^{d}\right)-\alpha\left(1-m_{i j}^{d}\right)>0$. Hence, $\left(m_{j i}^{d}, m_{i j}^{d}\right) \in M_{i}$. A symmetric argument works for the second part of the lemma.

Lemma A2: Suppose that $m_{i j}^{d} \geq m_{j i}^{d}$. Then $\left(m_{i j}^{d}, m_{j i}^{d}\right) \in M$ if and only if $\left(m_{i j}^{d}, m_{j i}^{d}\right) \in M_{i}$.

Proof of Lemma A2: It is obvious that $\left(m_{i j}^{d}, m_{j i}^{d}\right) \in M$ implies $\left(m_{i j}^{d}, m_{j i}^{d}\right) \in$ $M_{i}$. So suppose that $\left(m_{i j}^{d}, m_{j i}^{d}\right) \in M_{i}$. Then by symmetry of the definitions of $M_{i}$ and $M_{j},\left(m_{j i}^{d}, m_{i j}^{d}\right) \in M_{j}$. By Lemma A1, $\left(m_{j i}^{d}, m_{i j}^{d}\right) \in M_{i}$. Applying symmetry of the definitions again yields $\left(m_{i j}^{d}, m_{j i}^{d}\right) \in M_{j}$. Hence $\left(m_{i j}^{d}, m_{j i}^{d}\right) \in M_{j} \cap M_{i}=M$.

Lemma A3: Suppose that $\left(m_{i j}^{d}, m_{j i}^{d}\right) \in M$. Then $\left(m_{j i}^{d}, m_{i j}^{d}\right) \in M$ and the line segment $\left[\left(m_{i j}^{d}, m_{j i}^{d}\right),\left(m_{j i}^{d}, m_{i j}^{d}\right)\right] \subseteq M$. In particular, if $M \neq \emptyset$, then it contains a point on the diagonal segment $[(0,0),(1,1)]$.

Proof of Lemma A3: First, if $\left(m_{i j}^{d}, m_{j i}^{d}\right) \in M$, then $\left(m_{j i}^{d}, m_{i j}^{d}\right) \in M$ by symmetry of the definitions of $M_{i}$ and $M_{j}$. Now consider the line segment $\left[\left(m_{i j}^{d}, m_{j i}^{d}\right),\left(m_{j i}^{d}, m_{i j}^{d}\right)\right]$. In particular, consider the case $m_{i j}^{d} \geq m_{j i}^{d}$ and the line segment between $\left(m_{i j}^{d}, m_{j i}^{d}\right)$ and the point $(m, m)$ on the diagonal, $\left[\left(m_{i j}^{d}, m_{j i}^{d}\right),(m, m)\right] \subseteq\left[\left(m_{i j}^{d}, m_{j i}^{d}\right),\left(m_{j i}^{d}, m_{i j}^{d}\right)\right]$ (the line segment $\left[(m, m),\left(m_{j i}^{d}, m_{i j}^{d}\right)\right]$ can be covered with a symmetric argument). Since for all $\left(\widehat{m}_{i j}^{d}, \widehat{m}_{j i}^{d}\right) \in\left[\left(m_{i j}^{d}, m_{j i}^{d}\right),(m, m)\right]$, 
$\widehat{m}_{i j}^{d} \geq \widehat{m}_{j i}^{d}$, by Lemma A2 it suffices to show that $\left(\widehat{m}_{i j}^{d}, \widehat{m}_{j i}^{d}\right) \in M_{i}$. We must verify the equation stating that $\left(\widehat{m}_{i j}^{d}, \widehat{m}_{j i}^{d}\right) \in M_{i}$, namely

$$
f\left(\widehat{m}_{i j}^{d}, \widehat{m}_{j i}^{d}\right)+h\left(\widehat{m}_{i j}^{d}, \widehat{m}_{j i}^{d}\right)-\alpha \cdot\left(1-\widehat{m}_{j i}^{d}\right)>0
$$

Now for all $\left(\widehat{m}_{i j}^{d}, \widehat{m}_{j i}^{d}\right) \in\left[\left(m_{i j}^{d}, m_{j i}^{d}\right),(m, m)\right]$, there exists an $x \geq 0$ with $\widehat{m}_{i j}^{d}=$ $m_{i j}^{d}-x \geq m_{j i}^{d}+x=\widehat{m}_{j i}^{d}$, since the line segment lies below the diagonal. Now

$$
\begin{aligned}
& f\left(m_{i j}^{d}-x, m_{j i}^{d}+x\right)-f\left(m_{i j}^{d}, m_{j i}^{d}\right) \\
= & \beta \cdot\left[\left(1-m_{j i}^{d}-m_{i j}^{d}\right) \cdot\left(m_{i j}^{d}-x\right) \cdot\left(m_{j i}^{d}+x\right)\right]^{\frac{1}{3}}-\beta \cdot\left[\left(1-m_{j i}^{d}-m_{i j}^{d}\right) \cdot\left(m_{i j}^{d}\right) \cdot\left(m_{j i}^{d}\right)\right]^{\frac{1}{3}} \\
= & \beta \cdot\left[\left(1-m_{j i}^{d}-m_{i j}^{d}\right) \cdot\left(m_{i j}^{d}\right) \cdot\left(m_{j i}^{d}\right)+\left(1-m_{j i}^{d}-m_{i j}^{d}\right) \cdot x \cdot\left(m_{i j}^{d}-m_{j i}^{d}-x\right)\right]^{\frac{1}{3}} \\
& -\beta \cdot\left[\left(1-m_{j i}^{d}-m_{i j}^{d}\right) \cdot\left(m_{i j}^{d}\right) \cdot\left(m_{j i}^{d}\right)\right]^{\frac{1}{3}} \\
\geq & \beta \cdot\left[\left(1-m_{j i}^{d}-m_{i j}^{d}\right) \cdot\left(m_{i j}^{d}\right) \cdot\left(m_{j i}^{d}\right)+\left(1-m_{j i}^{d}-m_{i j}^{d}\right) \cdot x^{2}\right]^{\frac{1}{3}} \\
& -\beta \cdot\left[\left(1-m_{j i}^{d}-m_{i j}^{d}\right) \cdot\left(m_{i j}^{d}\right) \cdot\left(m_{j i}^{d}\right)\right]^{\frac{1}{3}} \\
\geq & 0 \\
& \\
& h\left(m_{i j}^{d}-x, m_{j i}^{d}+x\right)-h\left(m_{i j}^{d}, m_{j i}^{d}\right) \\
= & \gamma \cdot\left[\left(m_{j i}^{d}+x\right) \cdot\left(1-m_{j i}^{d}-m_{i j}^{d}\right)\right]^{\frac{1}{2}}-\gamma \cdot\left[m_{j i}^{d} \cdot\left(1-m_{j i}^{d}-m_{i j}^{d}\right)\right]^{\frac{1}{2}} \geq 0
\end{aligned}
$$

Finally,

$$
\alpha \cdot\left(1-m_{j i}^{d}-x\right) \leq \alpha \cdot\left(1-m_{j i}^{d}\right)
$$

Hence,

$$
\begin{aligned}
& f\left(\widehat{m}_{i j}^{d}, \widehat{m}_{j i}^{d}\right)+h\left(\widehat{m}_{i j}^{d}, \widehat{m}_{j i}^{d}\right)-\alpha \cdot\left(1-\widehat{m}_{j i}^{d}\right) \\
= & f\left(m_{i j}^{d}-x, m_{j i}^{d}+x\right)+h\left(m_{i j}^{d}-x, m_{j i}^{d}+x\right)-\alpha \cdot\left(1-m_{j i}^{d}-x\right) \\
\geq & f\left(m_{i j}^{d}, m_{j i}^{d}\right)+h\left(m_{i j}^{d}, m_{j i}^{d}\right)-\alpha \cdot\left(1-m_{j i}^{d}\right)>0
\end{aligned}
$$

The last line follows because $\left(m_{i j}^{d}, m_{j i}^{d}\right) \in M$.

Lemma A4: For any constant $a \in(-1,1)$ the intersection of the set $M$ and the line $\left\{\left(m_{i j}^{d}, m_{j i}^{d}\right) \in \mathbb{R}_{+}^{2} \mid m_{i j}^{d}+m_{j i}^{d} \leq 1, m_{j i}^{d}=m_{i j}^{d}-a\right\}$ is a convex set.

Proof of Lemma A4: Since $M$ is symmetric with respect to the diagonal $m_{i j}^{d}=m_{j i}^{d}$, let us consider $a \geq 0$. Setting $m_{j i}^{d}=m_{i j}^{d}-a$ in (14), define

$$
\begin{aligned}
k\left(m_{i j}^{d}\right) \equiv & F_{i}\left(m_{i j}^{d}, m_{i j}^{d}-a\right) \\
= & \beta\left[\left(1+a-2 m_{i j}^{d}\right) m_{i j}^{d}\left(m_{i j}^{d}-a\right)\right]^{1 / 3} \\
& +\gamma\left[\left(1+a-2 m_{i j}^{d}\right)\left(m_{i j}^{d}-a\right)\right]^{1 / 2}-\alpha\left(1+a-m_{i j}^{d}\right)
\end{aligned}
$$

Since $m_{j i}^{d}=m_{i j}^{d}-a \geq 0$ and $1 \geq m_{i j}^{d}+m_{j i}^{d}=2 m_{i j}^{d}-a$, the domain of the function $k$ is

$$
a \leq m_{i j}^{d} \leq \frac{1+a}{2} \quad \text { where } \quad 0 \leq a<1
$$


By Lemma A2, the intersection of the set $M$ and the line $m_{j i}^{d}=m_{i j}^{d}-a$ is the set of points satisfying

$$
k\left(m_{i j}^{d}\right)>0 .
$$

We show that function $k\left(m_{i j}^{d}\right)$ is strictly concave on $\left(a, \frac{1+a}{2}\right)$, and thus the set of points satisfying the inequality is convex. Differentiation of the function $k$ yields

$$
k^{\prime}\left(m_{i j}^{d}\right)=A\left(m_{i j}^{d}\right)+B\left(m_{i j}^{d}\right)+\alpha
$$

where

$$
\begin{gathered}
A\left(m_{i j}^{d}\right) \equiv \frac{\beta}{3}\left[\left(1+a-2 m_{i j}^{d}\right) m_{i j}^{d}\left(m_{i j}^{d}-a\right)\right]^{-2 / 3}\left[-6\left(m_{i j}^{d}\right)^{2}+2 m_{i j}^{d}(1+3 a)-a(1+a)\right] \\
B\left(m_{i j}^{d}\right) \equiv \frac{\gamma}{2}\left[\left(1+a-2 m_{i j}^{d}\right)\left(m_{i j}^{d}-a\right)\right]^{-1 / 2}\left(1+3 a-4 m_{i j}^{d}\right)
\end{gathered}
$$

The second derivative of $k$ is

$$
k^{\prime \prime}\left(m_{i j}^{d}\right)=A^{\prime}\left(m_{i j}^{d}\right)+B^{\prime}\left(m_{i j}^{d}\right)
$$

where

$$
\begin{gathered}
A^{\prime}\left(m_{i j}^{d}\right)=-\frac{2 \beta\left\{\left(m_{i j}^{d}\right)^{2}\left(1+3 a^{2}\right)-a(1+a)(1+3 a) m_{i j}^{d}+a^{2}(1+a)^{2}\right\}}{9\left[\left(1+a-2 m_{i j}^{d}\right) m_{i j}^{d}\left(m_{i j}^{d}-a\right)\right]^{5 / 3}} \\
=-\frac{2 \beta\left\{\left[m_{i j}^{d}\left(1+3 a^{2}\right)-\frac{a(1+a)(1+3 a)}{2}\right]^{2}+\frac{3 a^{2}(1+a)^{2}(1-a)^{2}}{4}\right\}}{9\left[\left(1+a-2 m_{i j}^{d}\right) m_{i j}^{d}\left(m_{i j}^{d}-a\right)\right]^{5 / 3}\left(1+3 a^{2}\right)} \\
B^{\prime}\left(m_{i j}^{d}\right)=-\frac{\gamma(1-a)^{2}}{4\left[\left(1+a-2 m_{i j}^{d}\right)\left(m_{i j}^{d}-a\right)\right]^{3 / 2}}
\end{gathered}
$$

implying that $k^{\prime \prime}\left(m_{i j}^{d}\right)=A^{\prime}\left(m_{i j}^{d}\right)+B^{\prime}\left(m_{i j}^{d}\right)<0$ on $\left(a, \frac{1+a}{2}\right)$, so $k$ is strictly concave on $\left(a, \frac{1+a}{2}\right)$. Thus, $\left\{m_{i j}^{d} \in\left(a, \frac{1+a}{2}\right) \mid k\left(m_{i j}^{d}\right)>0\right\}$ is convex, and the proof of the lemma is complete.

Lemma A5: Every point in $M \cap((0,0),(1,1))$ has a neighborhood contained in $M$.

Proof of Lemma A5: This follows directly from the definition of $M$; it implies that $M$ is an open set.

Theorem A2 follows directly from the combination of all of the Lemmata in this section. 


\subsection{Appendix c}

Lemma A6: The function $g(m)$ defined by (20) has the following properties:

(i) $g(m)$ is strictly quasi-concave on $\left[0, \frac{1}{2}\right]$.

(ii) $g(m)$ achieves its maximal value at $m^{B} \in\left[\frac{1}{3}, \frac{2}{5}\right]$.

(iii) The point $\left(m^{B}, m^{B}\right)$ corresponds to the bliss point $B$ in Figure 2, which is the unique point contained in every $M$ that is nonempty.

Proof of Lemma A6: (i) and (ii): For $m \in\left[0, \frac{1}{2}\right]$, let

$$
x(m) \equiv \frac{m}{1-m} \text { or } m(x)=\frac{x}{1+x}
$$

and define

$$
G(x) \equiv \beta\left[(1-x) x^{2}\right]^{1 / 3}+\gamma[(1-x) x]^{1 / 2} \text { for } x \in[0,1]
$$

Then, using definition (20)

$$
g(m)=G(x(m))
$$

Hence,

$$
g^{\prime}(m)=G^{\prime}(x(m)) \cdot x^{\prime}(m)
$$

Notice that

$$
x^{\prime}(m)=1+\frac{m}{(1-m)^{2}}>0
$$

so

$$
g^{\prime}(m) \gtreqless 0 \text { exactly as } G^{\prime}(x(m)) \gtreqless 0 \text {. }
$$

Now

$$
G^{\prime}(x)=C(x)+D(x)
$$

where

$$
\begin{aligned}
& C(x) \equiv \frac{\beta}{3}\left[(1-x) x^{2}\right]^{-2 / 3}(2-3 x) x \\
& D(x) \equiv \frac{\gamma}{2}[(1-x) x]^{-1 / 2}(1-2 x)
\end{aligned}
$$

Taking the derivatives of $C$ and $D$ respectively yields

$$
\begin{aligned}
& C^{\prime}(x)=-\frac{2 \beta}{9}(1-x)^{-5 / 3} x^{-4 / 3}<0 \\
& D^{\prime}(x)=-\frac{\gamma}{4}(1-x)^{-3 / 2} x^{-3 / 2}<0
\end{aligned}
$$

Therefore, considering that

$$
\begin{aligned}
& C(x) \gtreqless 0 \text { as } x \lesseqgtr 2 / 3 \\
& D(x) \gtreqless 0 \text { as } x \lesseqgtr 1 / 2
\end{aligned}
$$


we can conclude that there exists a unique $x^{*} \in[1 / 2,2 / 3]$ such that

$$
G^{\prime}(x) \gtreqless 0 \text { as } x \lesseqgtr x^{*}
$$

meaning that $G$ is strictly single peaked and strictly quasi-concave, achieving its maximum value exactly at $x^{*}$. Hence, the function $g(m)$ also is strictly single peaked and strictly quasi-concave, achieving its maximum value at

$$
m^{B} \equiv m\left(x^{*}\right)=\frac{x^{*}}{1+x^{*}} \in[1 / 3,2 / 5]
$$

(iii) To show that the point $\left(m^{B}, m^{B}\right)$ corresponds to the bliss point $B$ in Figure 2, let us recall how the bliss point has been defined. Let $M(\alpha)$ be the set $M$ under the parameter value $\alpha>0$. Then, a point $\left({ }^{*} m_{i j}^{d},{ }^{*} m_{j i}^{d}\right) \in \mathbb{R}^{2}$ is called a bliss point if it holds that for any $\alpha>0$,

$$
M(\alpha) \neq \emptyset \Longrightarrow\left({ }^{*} m_{i j}^{d}{ }^{*} m_{j i}^{d}\right) \in M(\alpha)
$$

To show the existence and the uniqueness of such a point, since $M(\alpha)$ is symmetric to the diagonal, let us focus on the lower half of $M(\alpha)$, and define

$$
M^{L}(\alpha)=\left\{\left(m_{i j}^{d}, m_{j i}^{d}\right) \in M(\alpha) \mid m_{i j}^{d} \geq m_{j i}^{d}\right\}
$$

Then, by Lemma A2, $M^{L}(\alpha)$ coincides with the lower part of $M_{i}$ associated with $\alpha$ :

$$
\begin{aligned}
M^{L}(\alpha) & =\left\{\left(m_{i j}^{d}, m_{j i}^{d}\right) \in M_{i}(\alpha) \mid m_{i j}^{d} \geq m_{j i}^{d}\right\} \\
& =\left\{\left(m_{i j}^{d}, m_{j i}^{d}\right) \in \mathbb{R}^{2} \mid m_{i j}^{d} \geq m_{j i}^{d} \geq 0, m_{i j}^{d}+m_{j i}^{d} \leq 1,\right. \\
& \left.f\left(m_{i j}^{d}, m_{j i}^{d}\right)+h\left(m_{i j}^{d}, m_{j i}^{d}\right)-\alpha\left(1-m_{j i}^{d}\right)>0\right\}
\end{aligned}
$$

When $m_{i j}^{d}+m_{j i}^{d}=1$ or $m_{j i}^{d}=0$, we have $f\left(m_{i j}^{d}, m_{j i}^{d}\right)=h\left(m_{i j}^{d}, m_{j i}^{d}\right)=0$, implying that $M^{L}(\alpha)$ does not contain any point $\left(m_{i j}^{d}, m_{j i}^{d}\right)$ such that $m_{i j}^{d}+$ $m_{j i}^{d}=1$ or $m_{j i}^{d}=0$. Thus, we can rewrite $M^{L}(\alpha)$ as follows:

$$
\begin{aligned}
M^{L}(\alpha) & =\left\{\left(m_{i j}^{d}, m_{j i}^{d}\right) \in \mathbb{R}^{2} \mid m_{i j}^{d} \geq m_{j i}^{d}>0, m_{i j}^{d}+m_{j i}^{d}<1, \frac{f\left(m_{i j}^{d}, m_{j i}^{d}\right)}{1-m_{j i}^{d}}+\frac{h\left(m_{i j}^{d}, m_{j i}^{d}\right)}{1-m_{j i}^{d}}>\alpha\right\} \\
& =\left\{\left(m_{i j}^{d}, m_{j i}^{d}\right) \in \mathbb{R}^{2} \mid m_{i j}^{d} \geq m_{j i}^{d}>0, m_{i j}^{d}+m_{j i}^{d}<1,\right. \\
& \left.\beta\left[\left(1-\frac{m_{i j}^{d}}{1-m_{j i}^{d}}\right) \frac{m_{i j}^{d}}{1-m_{j i}^{d}} \frac{m_{j i}^{d}}{1-m_{j i}^{d}}\right]^{1 / 3}+\gamma\left[\left(1-\frac{m_{i j}^{d}}{1-m_{j i}^{d}}\right) \frac{m_{j i}^{d}}{1-m_{j i}^{d}}\right]^{1 / 2}>\alpha\right\}
\end{aligned}
$$

Given any $\left(m_{i j}^{d}, m_{j i}^{d}\right) \in M^{L}(\alpha)$ such that $m_{i j}^{d}>m_{j i}^{d}$, define

$$
m \equiv \frac{m_{i j}^{d}+m_{j i}^{d}}{2}
$$


Then, $m_{i j}^{d}>m>m_{j i}^{d}$, and $(m, m) \in M^{L}(\alpha)$ by Lemma A3. Furthermore,

$$
\begin{aligned}
& \left(1-\frac{m}{1-m}\right)\left(\frac{m}{1-m}\right)^{2}-\left(1-\frac{m_{i j}^{d}}{1-m_{j i}^{d}}\right) \frac{m_{i j}^{d}}{1-m_{j i}^{d}} \frac{m_{j i}^{d}}{1-m_{j i}^{d}} \\
= & \frac{\left(1-m_{i j}^{d}-m_{j i}^{d}\right) m^{2}}{(1-m)^{3}}-\frac{\left(1-m_{i j}^{d}-m_{j i}^{d}\right) m_{i j}^{d} m_{j i}^{d}}{\left(1-m_{j i}^{d}\right)^{3}} \\
> & \frac{\left(1-m_{i j}^{d}-m_{j i}^{d}\right)}{\left(1-m_{j i}^{d}\right)^{3}}\left(m^{2}-m_{i j}^{d} m_{j i}^{d}\right) \\
= & \frac{\left(1-m_{i j}^{d}-m_{j i}^{d}\right)}{\left(1-m_{j i}^{d}\right)^{3}} \frac{\left(m_{i j}^{d}-m_{j i}^{d}\right)^{2}}{4}>0
\end{aligned}
$$

Likewise,

$$
\begin{aligned}
& \left(1-\frac{m}{1-m}\right) \frac{m}{1-m}-\left(1-\frac{m_{i j}^{d}}{1-m_{j i}^{d}}\right) \frac{m_{j i}^{d}}{1-m_{j i}^{d}} \\
= & \frac{\left(1-m_{i j}^{d}-m_{j i}^{d}\right) m}{(1-m)^{2}}-\frac{\left(1-m_{i j}^{d}-m_{j i}^{d}\right) m_{j i}^{d}}{\left(1-m_{j i}^{d}\right)^{2}} \\
> & \frac{\left(1-m_{i j}^{d}-m_{j i}^{d}\right)}{\left(1-m_{j i}^{d}\right)^{2}}\left(m-m_{j i}^{d}\right)>0
\end{aligned}
$$

Therefore, using the function $g(m)$ defined by (20), we can conclude that when $m_{i j}^{d}>m_{j i}^{d}$ and $m \equiv\left(m_{i j}^{d}+m_{j i}^{d}\right) / 2$,

$g(m)>\beta\left[\left(1-\frac{m_{i j}^{d}}{1-m_{j i}^{d}}\right) \frac{m_{i j}^{d}}{1-m_{j i}^{d}} \frac{m_{j i}^{d}}{1-m_{j i}^{d}}\right]^{1 / 3}+\gamma\left[\left(1-\frac{m_{i j}^{d}}{1-m_{j i}^{d}}\right) \frac{m_{j i}^{d}}{1-m_{j i}^{d}}\right]^{1 / 2}$

Moreover, (i) and (ii) of Lemma A6 mean that

$$
g\left(m^{b}\right)>g(m) \text { for any } m \neq m^{b}
$$

Combining (28), (29) and (30), we can conclude that given any $\left(m_{i j}^{d}, m_{j i}^{d}\right)$ such that $m_{i j}^{d} \geq m_{j i}^{d}$

$$
\left(m_{i j}^{d}, m_{j i}^{d}\right) \in M^{L}(\alpha) \Longrightarrow\left(m^{B}, m^{B}\right) \in M^{L}(\alpha) .
$$

That is,

$$
M^{L}(\alpha) \neq \emptyset \Longrightarrow\left(m^{B}, m^{B}\right) \in M^{L}(\alpha)
$$

Hence, the point $\left(m^{B}, m^{B}\right)$ is a bliss point. Finally, to show that the bliss point is unique, take any $\bar{\alpha}>0$ such that $M^{L}(\bar{\alpha}) \neq \emptyset$, and take any $\left(\bar{m}_{i j}^{d}, \bar{m}_{j i}^{d}\right) \in$ $M^{L}(\bar{\alpha})$ such that $\left(\bar{m}_{i j}^{d}, \bar{m}_{j i}^{d}\right) \neq\left(m^{B}, m^{B}\right)$. If $\bar{m}_{i j}^{d}>\bar{m}_{j i}^{d}$, then the inequality 
(29) holds when $\left(m_{i j}^{d}, m_{j i}^{d}\right)$ is replaced with $\left(\bar{m}_{i j}^{d}, \bar{m}_{j i}^{d}\right)$. If $\bar{m}_{i j}^{d}=\bar{m}_{j i}^{d}$, then $g\left(m^{B}\right)>g\left(\bar{m}_{i j}^{d}\right)$ by $(30)$. Hence, if we define

$\varepsilon \equiv g\left(m^{B}\right)-\left\{\beta\left[\left(1-\frac{\bar{m}_{i j}^{d}}{1-\bar{m}_{j i}^{d}}\right) \frac{\bar{m}_{i j}^{d}}{1-\bar{m}_{j i}^{d}} \frac{\bar{m}_{j i}^{d}}{1-\bar{m}_{j i}^{d}}\right]^{1 / 3}+\gamma\left[\left(1-\frac{\bar{m}_{i j}^{d}}{1-\bar{m}_{j i}^{d}}\right) \frac{\bar{m}_{j i}^{d}}{1-\bar{m}_{j i}^{d}}\right]^{1 / 2}\right\}$

then $\varepsilon$ is positive. Replacing $\alpha$ with $g\left(m^{B}\right)-\frac{\varepsilon}{2}$ and $\left(m_{i j}^{d}, m_{j i}^{d}\right)$ with $\left(\bar{m}_{i j}^{d}, \bar{m}_{j i}^{d}\right)$ in (28), we can see that

$$
\left(\bar{m}_{i j}^{d}, \bar{m}_{j i}^{d}\right) \notin M^{L}\left(g\left(m^{B}\right)-\frac{\varepsilon}{2}\right)
$$

whereas $\left(m^{B}, m^{B}\right) \in M^{L}\left(g\left(m^{B}\right)-\frac{\varepsilon}{2}\right)$. Thus, the point $\left(\bar{m}_{i j}^{d}, \bar{m}_{j i}^{d}\right)$ is not contained in the nonempty set $M^{L}\left(g\left(m^{B}\right)-\frac{\varepsilon}{2}\right)$, implying that the point $\left(\bar{m}_{i j}^{d}, \bar{m}_{j i}^{d}\right) \neq$ $\left(m^{B}, m^{B}\right)$ is not a bliss point. 